\title{
The Subluminous Supernova 2007qd: A Missing Link in a Family of Low-Luminosity Type Ia Supernovae
}

\author{
Colin M. M ${ }^{c}$ Clelland ${ }^{1}$, Peter M. Garnavich ${ }^{1}$, Lluís Galbany ${ }^{2}$, Ramon Miquel ${ }^{2,3}$, Ryan J. \\ Foley $^{4,5}$, Alexei V. Filippenko ${ }^{6}$, Bruce Bassett ${ }^{7}$, J. Craig Wheeler ${ }^{8}$, Ariel Goobar ${ }^{9}, 10$, \\ Saurabh W. Jha ${ }^{11}$, Masao Sako ${ }^{12}$, Joshua A. Frieman ${ }^{13,14,15}$, Jesper Sollerman ${ }^{16,17}$, Jozsef \\ Vinko $^{18}$, and Donald P. Schneider ${ }^{19}$
}




\begin{abstract}
We present multi-band photometry and multi-epoch spectroscopy of the peculiar Type Ia supernova (SN Ia) 2007qd, discovered by the SDSS-II Supernova Survey. It possesses physical properties intermediate to those of the peculiar
\end{abstract}

${ }^{1}$ Department of Physics, University of Notre Dame, Notre Dame, IN 46556.

${ }^{2}$ Institut de Física d'Altes Energies, Universitat Autònoma de Barcelona, E-08193 Barcelona, Spain.

${ }^{3}$ Institució Catalana de Recerca i Estudis Avançats, E-08010 Barcelona, Spain.

${ }^{4}$ Harvard-Smithsonian Center for Astrophysics, 60 Garden Street, Cambridge, MA 02138.

${ }^{5}$ Clay Fellow.

${ }^{6}$ Department of Astronomy, University of California, Berkeley, CA 94720-3411.

${ }^{7}$ Department of Mathematics and Applied Mathematics, University of Cape Town, Rondebosch 7701, South Africa.

${ }^{8}$ Department of Astronomy, McDonald Observatory, University of Texas, Austin, TX 78712.

${ }^{9}$ Department of Physics, Stockholm University, AlbaNova University Center, SE-106 91 Stockholm, Sweden.

${ }^{10}$ The Oskar Klein Centre for Cosmoparticle Physics, Department of Physics, AlbaNova, Stockholm University, SE-106 91 Stockholm, Sweden.

${ }^{11}$ Department of Physics and Astronomy, Rutgers University, 136 Frelinghuysen Road, Piscataway, NJ 08854 .

${ }^{12}$ Department of Physics and Astronomy, University of Pennsylvania, 209 South 33rd Street, Philadelphia, PA 19104.

${ }^{13}$ Kavli Institute for Cosmological Physics, University of Chicago, 5640 South Ellise Avenue, Chicago, IL 60637 .

${ }^{14}$ Department of Astronomy and Astrophysics, University of Chicago, 5640 South Ellise Avenue, Chicago, IL 60637.

${ }^{15}$ Center for Particle Astrophysics, Fermi National Accelerator Laboratory, P. O. Box 500, Batavia, IL 60510 .

${ }^{16}$ Dark Cosmology Centre, Niels Bohr Institute, University of Copenhagen, Juliane Maries Vej 30, DK-2100 Copenhagen, Denmark.

${ }^{17}$ Department of Astronomy, The Oskar Klein Centre, Stockholm University, 10691 Stockholm, Sweden.

${ }^{18}$ Department of Optics and Quantum Electronics, University of Szeged, Hungary.

${ }^{19}$ Department of Astronomy and Astrophysics, 525 Davey Laboratory, Pennsylvania State University, University Park, PA 16802. 
SN 2002cx and the extremely low-luminosity SN 2008ha. Optical photometry indicates that it had an extraordinarily fast rise time of $\lesssim 10$ days and a peak absolute $B$ magnitude of $-15.4 \pm 0.2$ at most, making it one of the most subluminous SN Ia ever observed. Follow-up spectroscopy of SN 2007qd near maximum brightness unambiguously shows the presence of intermediate-mass elements which are likely caused by carbon/oxygen nuclear burning. Near maximum brightness, SN 2007qd had a photospheric velocity of only $2800 \mathrm{~km} \mathrm{~s}^{-1}$, similar to that of SN 2008ha but about 4000 and $7000 \mathrm{~km} \mathrm{~s}^{-1}$ less than that of SN 2002cx and normal SN Ia, respectively. We show that the peak luminosities of SN 2002cx-like objects are highly correlated with both their light-curve stretch and photospheric velocities. Its strong apparent connection to other SN 2002cxlike events suggests that SN 2007qd is also a pure deflagration of a white dwarf, although other mechanisms cannot be ruled out. It may be a critical link between SN 2008ha and the other members of the SN 2002cx-like class of objects.

Subject headings: supernovae: general - supernovae: individual (SN 2007qd, SN 2008ha, SN 2002cx, SN 2005hk) — galaxies: individual (SDSS J020932.73005959.8)

\section{Introduction}

For decades, Type Ia supernovae (SN Ia) have been interpreted as thermonuclear explosions that result from either accretion onto a degenerate white dwarf or via coalescence of two degenerate stars; see Livio (2000) for a thorough review. Models that begin fusion subsonically and then turn into a detonation are best at matching observations of typical SN Ia (Khokhlov 1991). Synthesized intermediate-mass elements (IMEs) like silicon and sulfur in SN Ia spectra are the result of $\mathrm{C}$ and $\mathrm{O}$ burning at low densities due to the pre-expansion of the white dwarf during the deflagration phase. The deflagration turns into a detonation, and the combination of the two produces a significant amount of ${ }^{56} \mathrm{Ni}$, the decay of which powers the light curve.

SN Ia show a range of energies and spectral characteristics, but the majority of them are quite homogeneous when compared with other supernova types. The relative uniformity amongst SN Ia explosions has allowed their exploitation as cosmic standard candles, and observed correlations between light-curve shape, peak luminosity (Phillips 1993; Hamuy et al. 1996a), and color (Riess et al. 1996; Guy et al. 2007) have enabled the precise measurement of cosmologically interesting distances. It is with these measurements, taken over a range of redshifts, that estimates of the Hubble parameter $\left(H_{0}\right)$ and cosmic 
matter density have been refined (Hamuv et al. 1995; Riess et al. 1995; Garnavich et al. 1998; Freedman et al. 2001; Riess et al. 2009), and evidence for dark energy was revealed (Riess et al. 1998; Perlmutter et al. 1999).

The Sloan Digital Sky Survey-II (SDSS-II) Supernova Survey was designed to further improve SN Ia as distance indicators (Frieman et al. 2008; Sako et al. 2008). Covering a $300 \mathrm{deg}^{2}$ area with a rapid cadence, the survey discovered and spectroscopically confirmed roughly $500 \mathrm{SN}$ Ia over a range of redshifts out to $z \approx 0.4$. Kessler et al. (2009a), Lampeitl et al. (2009), and Sollerman et al. (2009) used the first year of SDSS-II data to constrain the dark energy equation-of-state parameter $w=P /\left(\rho c^{2}\right)$, and analyzed the systematic errors limiting the cosmological measurements.

Identifying the progenitors of SN Ia and better understanding their explosion mechanism may improve their reliability as distance indicators. One approach is to study events that do not conform to the general SN Ia homogeneity in their spectra or luminosity. Subclasses of SN 1991T-like (hereafter referred to as 91T; Filippenko et al. 1992b; Phillips et al. 1992) and SN 1991bg-like (hereafter 91bg; Filippenko et al. 1992a; Leibundgut et al. 1993; Turatto et al. 1996; Garnavich et al. 2004) events were quickly recognized as being peculiar, although much of the spectroscopic diversity is now known to be caused by a range of photospheric temperatures (Nugent et al. 1995). Recently, additional subclasses of SN Ia have been identified. For example, SN 2002ic (Hamuy et al. 2003) and SN 2005gj (Prieto et al. 2005; Aldering et al. 2006) are luminous objects that show hydrogen emission lines in their spectra, unlike normal SN Ia. It is suspected they may be SN Ia interacting with dense circumstellar material, although a core-collapse scenario has also been proposed (Benetti et al. 2006).

SN 2002cx (hereafter 02cx) was especially peculiar (Filippenko 2003; Li et al. 2003). It showed a hot (91T-like) spectrum at early times, but it cooled quickly after maximum brightness and its expansion velocities were well below typical for a SN Ia. 02cx deviated significantly from the Phillips (1993) relation between peak luminosity and decline rate; it was subluminous for its light-curve shape by $\sim 1.8 \mathrm{mag}$ in the $B$ and $V$ bands. The wellobserved SN 2005hk (hereafter 05hk) was spectroscopically very similar to $02 \mathrm{cx}$ - both exhibited photospheric velocities of roughly $7000 \mathrm{~km} \mathrm{~s}^{-1}$. Photometrically, $05 \mathrm{hk}$ was faint on the Phillips relation by $\sim 0.6-1.0 \mathrm{mag}$ in the optical, but $\sim 0.5$ mag more luminous than 02cx (Phillips et al. 2007).

Jha et al. (2006) have identified several other 02cx-like objects and postulated that their extreme subluminosity suggests that they constitute a class of pure thermonuclear deflagrations: that is, the fusion front moving through the white dwarf fails to make the transition to supersonic burning (Branch et al. 2004) and is unable to generate the large amounts of 
radioactive nickel observed in typical SN Ia (Foley et al. 2009). The thermonuclear burning of carbon and oxygen at moderate densities will create IMEs such as silicon, sulfur, and calcium that dominate the spectrum. Though typical SN Ia experience this phase only briefly, Jha et al. (2006) reasoned that 02cx-like objects may burn completely via this mechanism. (For a list of 02cx-like events, see Table 9 of Foley et al. 2009.)

Which characteristics separate delayed detonations from pure deflagrations? Simulations of pure deflagration events predict that Rayleigh-Taylor instabilities will form clumps of radioactive nickel and mix unburned material toward the center (Reinecke et al. 2002; Gamezo et al. 2004; Travaglio et al. 2004; Schmidt \& Niemeyer 2006). Late-time spectra of 02cx-like events show many permitted iron transitions broken up into thin spikes (Jha et al. 2006), indicative of the lower velocities that characterize 02cx-like supernovae. These spectra also show narrow forbidden lines of Ca II and Fe II, but are quite distinct from normal SN Ia at late epochs, whose spectra are dominated by relatively broad, blended Fe II and Fe III lines. Spectra of $05 \mathrm{hk} \gtrsim 200$ days past maximum have been analyzed for emission from unburned C I and O I, but their existence has not been confirmed (Sahu et al. 2008).

An extremely subluminous transient, SN 2008ha (hereafter 08ha; Folev et al. 2009), appears to be an additional member of the 02cx class, although Valenti et al. (2009) have proposed that the extreme nature of 08ha (and other 02cx-like objects) is better matched by the core collapse of a massive star where most of the synthesized radioactive elements fall back to a black hole. With the discovery of Si II and S II in the early-time spectra of 08ha (Foley et al. 2010), that proposition becomes less likely.

Here, we report photometry and spectroscopy of SN 2007qd (hereafter 07qd). Its observed properties place it in the 02cx subclass of supernovae, specifically as a member intermediate to 02cx and 08ha, linking these objects. We present the photometric and spectroscopic observations of $07 \mathrm{qd}$ and compare its unique properties with a range of other SN. We estimate the rise time, luminosity distance, and peak luminosity of this peculiar object, and we calculate its kinetic energy. Furthermore, we model the spectra to determine the velocity and composition of the ejecta and compare these with the findings of other similar SN to identify common traits. With this information we predict the synthesized ${ }^{56} \mathrm{Ni}$ mass and determine whether 07qd shares more in common with a thermonuclear deflagration or a core-collapse scenario. 


\section{Observations}

\subsection{Photometry}

07qd (Bassett et al. 2007) was discovered during the SDSS-II Supernova Survey using the SDSS Camera on the $2.5 \mathrm{~m}$ telescope (Gunn et al. 1998, 2006) at Apache Point Observatory. The $\mathrm{SN}$ was located amidst a spiral arm at $\alpha=02^{h} 09^{m} 33.56^{s}, \delta=-01^{\circ} 00^{\prime} 02.2^{\prime \prime}$ (J2000.0), a projected distance of $10.6 \mathrm{kpc}$ from the nucleus of the SBb/SBc host galaxy SDSS J020932.73-005959.8 centered at $\alpha=02^{h} 09^{m} 32.73^{s}, \delta=-00^{\circ} 59^{\prime} 59.80^{\prime \prime}$ (Bassett et al. 2007). The redshift of the host is $z=0.043147 \pm 0.00004$, as measured from the SDSS galaxy redshift survey (York et al. 2000; Adelman-McCarthy et al. 2008). Figure 1 shows an image of $07 \mathrm{qd}$ and its location in its host galaxy. The foreground Milky Way extinction in the direction of $07 \mathrm{qd}$ is $E(B-V)=0.035 \mathrm{mag}$ as calculated from the dust maps of Schlegel et al. (1998).

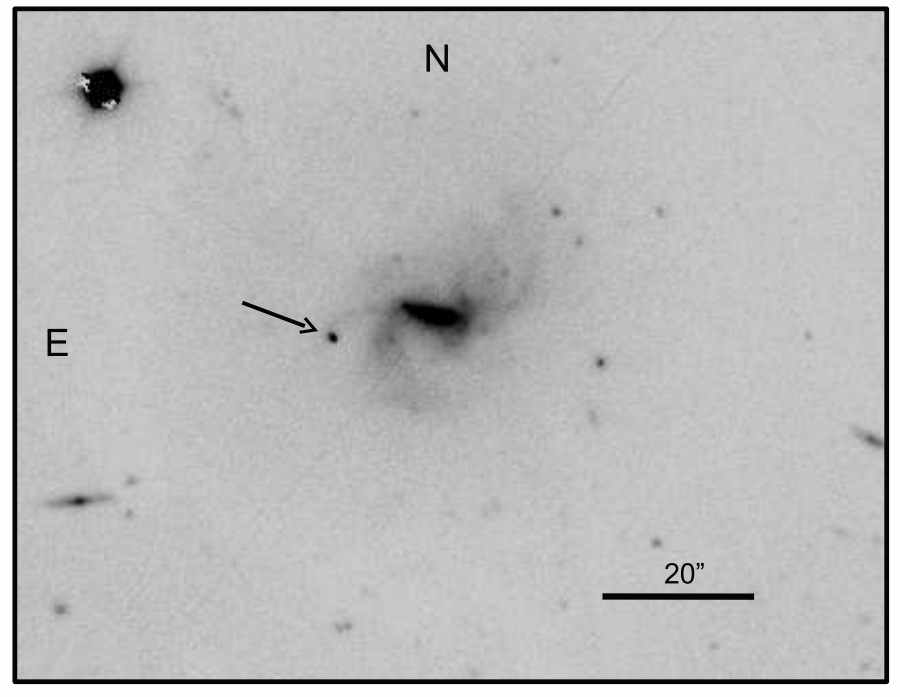

Fig. 1. - Image of $07 q d$ (denoted by arrow) relative to its host galaxy in a $105^{\prime \prime} \times 80^{\prime \prime}$ window . The 90-s unfiltered exposure was taken on MJD 54409.08 with the TNG telescope.

The photometry was calibrated in the standard SDSS ugriz photometric system (Fukugita et al. 1996; Smith et al. 2002). The flux from the supernova was estimated by using the scene modeling technique (Holtzman et al. 2008) from individual calibrated images and without spatial resampling. Figure 2 shows the SDSS-II light curves in the ugriz bands; the data are listed in Table 1. The time of peak bolometric flux for 07qd is not well defined, but probably 
occurred within a span of 2 days around maximum apparent $g$-band magnitude $20.69 \pm 0.06$ mag on MJD 54405.39. We base this on both the available $u, g$, and $r$ data detected on that date, and the fact that the $u$ and $g$ fluxes were falling while the $r, i$, and $z$ fluxes were still rising.

Bassett et al. (2007) noted that the spectrum of 07qd was similar to that of 05hk, so we compared their light curves. While the maximum $g$-band apparent magnitude of $05 \mathrm{hk}$ was $g=16.32 \pm 0.02$, it achieved a peak $B$-band absolute magnitude of $M_{B}=-18.0 \pm 0.3$. This is rather $\operatorname{dim}$ for a typical SN Ia $\left(M_{B} \approx-19 \mathrm{mag}\right)$, but $\sim 2.6 \mathrm{mag}$ brighter than $07 \mathrm{qd}$ (see $\S 3.1$ ). Featured in Figure 2 are the 05hk light curves corrected to the observed frame of 07qd. In addition, the brightness of $05 \mathrm{hk}$ has been reduced by 2.4 mag to better match the peak flux of $07 \mathrm{qd}$ and enable their shapes to be compared. Ignoring possible differences in dust extinction, 05hk is more luminous than 07qd in all of the SDSS bands, and 05hk both rose and declined more slowly in $u, g$, and $r$ than $07 \mathrm{qd}$. The light-curve widths of the two supernovae are similar at near-infrared wavelengths (SDSS $i, z)$, though 07qd shows a very slow decline rate at red wavelengths. 05hk also appears to peak later than $07 \mathrm{qd}$ as the bandpass becomes progressively redder.

We compared the light curve of 07qd with those of additional supernovae to identify any possibly similarity to the Type Ic or II classes. As 07qd rose rather quickly, we compared it to other events which shared similar rise times. We chose the fast-evolving SN 1994I (Yokoo et al. 1994; Richmond et al. 1996; Sauer et al. 2006), a well-observed prototype for SN Ic not associated with a gamma-ray burst, and SN 1999gi (Leonard et al. 2002), a Type IIP (plateau) SN whose absolute $B$ magnitude at peak was similar to that of 07qd (described in detail in $\S 3.1$ ). Figure 3 exhibits the shapes of these light curves in the $r$ band. Though the steep rise implied by the 07qd data may be similar to those of a SN IIP or SN Ic, the decline of 07qd and 05hk fails to represent the steep drop of SN 1994I or the extended plateau of SN 1999gi. In addition, the rise of 07qd is far faster than that of 05hk and is more similar to that of 08ha (Foley et al. 2009, 2010).

Figure 4 compares the color evolution of $07 \mathrm{qd}$ to that of $05 \mathrm{hk}$, and reveals the former to be more blue near maximum brightness. The colors of normal SN Ia are fairly well established (Phillips et al. 1999; Riess et al. 1996) and are used to estimate the reddening caused by dust in the host galaxy. However, the intrinsic colors of peculiar 02cx-like events are uncertain, making the estimation of host-galaxy extinction problematic. 07qd is bluer than $05 \mathrm{hk}$, having lower $g-r$ colors of $0.21 \pm 0.09 \mathrm{mag}$ and $0.13 \pm 0.06 \mathrm{mag}$ at 0.4 and 1.3 days past $B$ maximum, respectively. This suggests that dust extinction is not the major cause of the low luminosity of 07qd compared with 05hk. Additionally, 07qd appears to decline slower in the $i$ and $z$ light curves. 

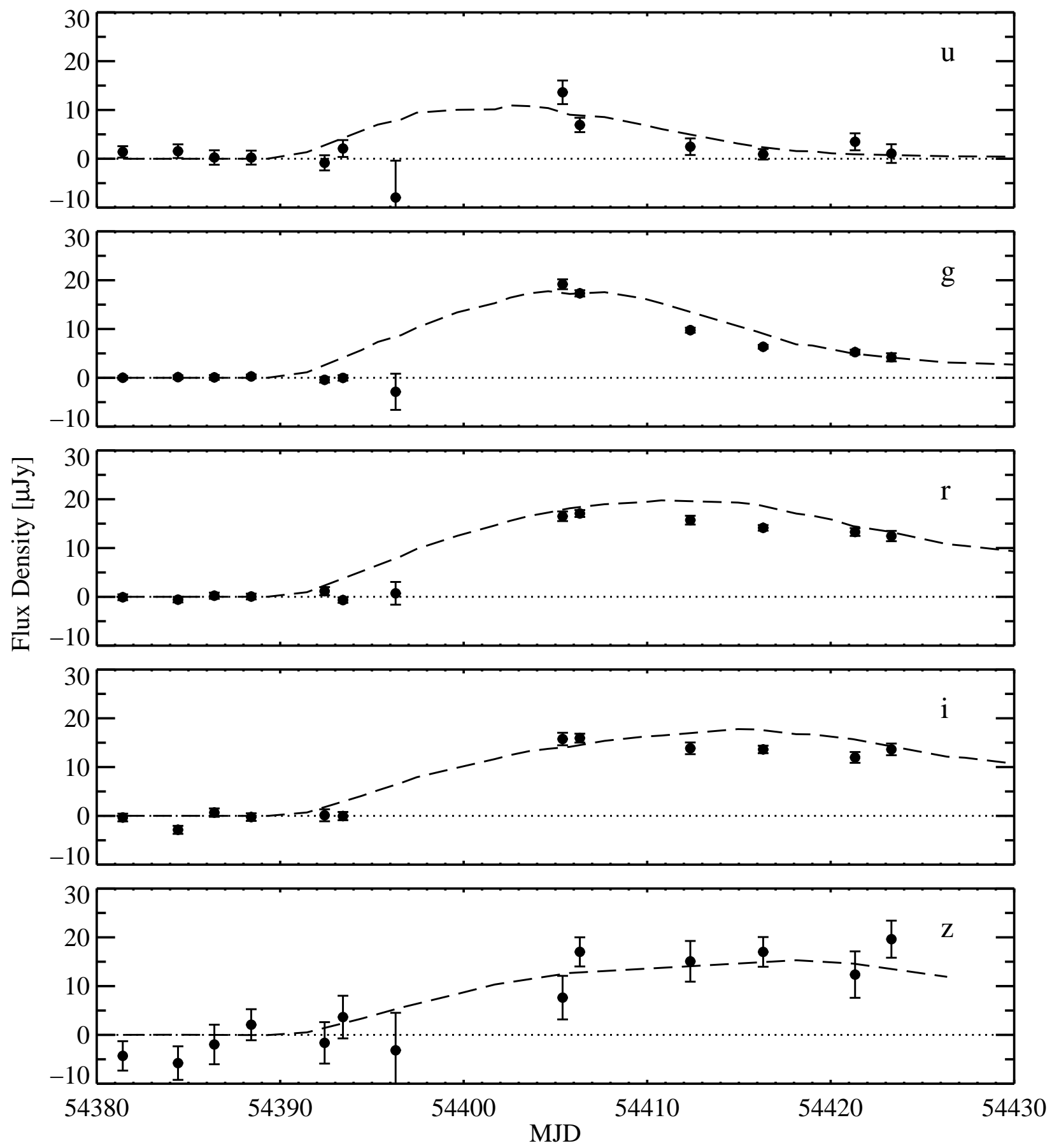

Fig. 2.- SDSS apparent light curves of 07qd given in flux-density units. The dashed line represents the combined SDSS and CSP light-curve data of 05hk in the observed frame of $07 \mathrm{qd}$, positioned over the maximum brightness of 07qd, and artificially shown 2.4 mag fainter for better comparison of their shapes. With respect to the early nondetections, 07qd clearly rose faster than $05 \mathrm{hk}$ in the $u, g, r$, and $i$ bands. Neither light curve has been corrected for host-galaxy extinction. 


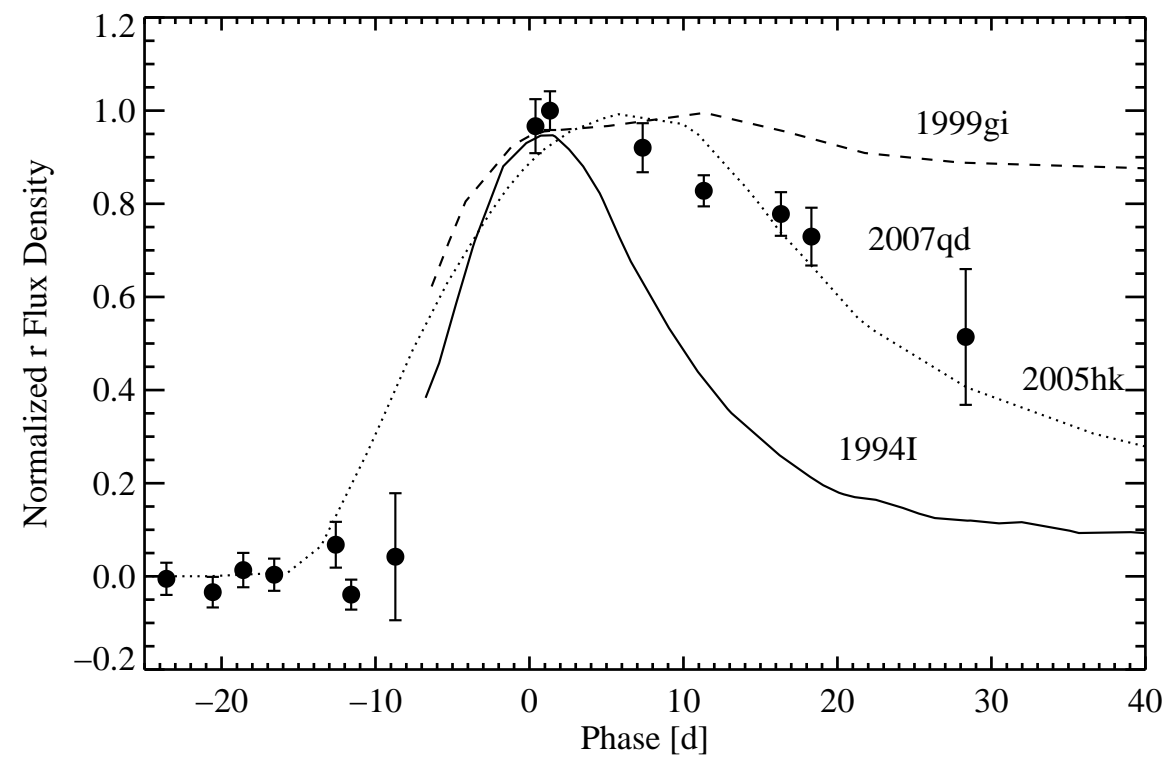

Fig. 3.- $r$-band light curves of SN 1994I (Type Ic, solid line), SN 1999gi (Type IIP, dashed line), 05hk (dotted line), and 07qd (data points) given in flux units. SN 1994I and SN 1999gi have been converted to the SDSS $r$ bandpass via conversions utilizing $V$ and $R$ by Fukugita et al. (1996), and all fluxes have been normalized to each maximum. The time axis has also been corrected to the rest frame, and each SN arbitrarily shifted in phase to best match the rise portion. SN 1994I, SN 1999gi, and 05hk are shown without error bars for clarity, and the interpolated segments were convolved with a Gaussian full width at half-maximum intensity (FWHM) of 2 days.

\subsection{Spectroscopy}

The $3.58 \mathrm{~m}$ Telescopio Nazionale Galileo (TNG) in the Canary Islands observed 07qd at 3 days after $B$ maximum (see $\S 3.1$ for the determination of $B_{\max }$ ). The spectrum is a composite of three 30-min exposures obtained with DOLORES (Device Optimized for LOw RESolution), a low-resolution spectrograph and camera permanently installed at the Nasmyth B focus of the TNG. It is equipped with a $2048 \times 2048$ pixel E2V 4240 thinned, back-illuminated, deep-depleted, Astro-BB coated CCD with a pixel size of $13.5 \mu \mathrm{m}$ and a field of view of $8.6^{\prime} \times 8.6^{\prime}$ with a $0.252^{\prime \prime}$ pixel $^{-1}$ scale. The spectra were observed with the low-resolution blue grism (LR-B, dispersion $2.52 \AA$ pixel $^{-1}$ ), covering the 3673-7401 range at an airmass of 1.27. A slit of $1.0^{\prime \prime}$ width, equal to the average seeing, was used for the observations and was aligned with the supernova and galaxy core at a position angle of $-39.3^{\circ}$.

The Hobby-Eberly Telescope (HET) at the McDonald Observatory in Texas collected 


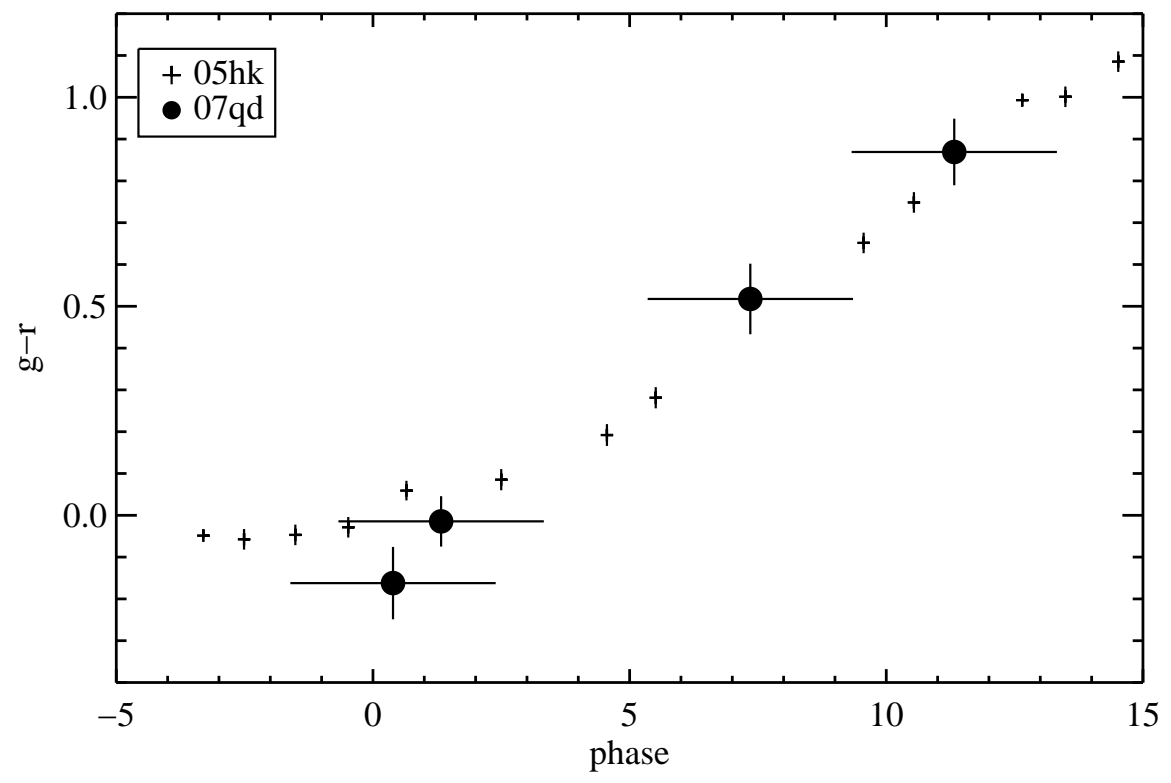

Fig. 4. - The $g-r$ color of $05 \mathrm{hk}$ and $07 \mathrm{qd}$ for the first two weeks past maximum brightness. The large uncertainty in the phase of 07qd stems from the 2-day uncertainty in the time of maximum. Neither SN has been corrected for reddening.

spectra of 20-min exposure times utilizing the Marcario Low Resolution Spectrograph (LRS; Hill et al. 1998) at 8 and 15 days past maximum. At the prime focus, the LRS employed a $0.235^{\prime \prime}$ pixel $^{-1}$ plate scale with a $1^{\prime \prime}$ wide by $4^{\prime}$ long slit and covered the 4075-9586 $\AA$ range, though low signal-to-noise ratios severely limit visibility past $8000 \AA$.

We obtained low-resolution spectra at 10 days past maximum brightness with the Low Resolution Imaging Spectrometer (LRIS; Oke et al. 1995) on the $10 \mathrm{~m}$ Keck I telescope on Mauna Kea, Hawaii. The Keck measurement was able to cover bluer wavelengths than the other observations, spanning the 3073-8800 A range. For all spectra, standard CCD processing and spectrum extraction were performed with IRAF1. The data were extracted using the optimal algorithm of Horne (1986). Low-order polynomial fits to calibration-lamp spectra were used to establish the wavelength scale, and small adjustments derived from night-sky lines in the object frames were applied. We employed our own IDL routines to flux calibrate the data and remove telluric lines using the well-exposed continua of spectrophotometric standard stars (Wade \& Horne 1988; Foley et al. 2003).

\footnotetext{
${ }^{1}$ IRAF: the Image Reduction and Analysis Facility is distributed by the National Optical Astronomy Observatory, which is operated by the Association of Universities for Research in Astronomy, Inc. (AURA) under cooperative agreement with the National Science Foundation (NSF).
} 
A journal of the spectroscopic observations is given in Table 2, and the resulting extracted and reduced spectra are detailed in Figure 5 .

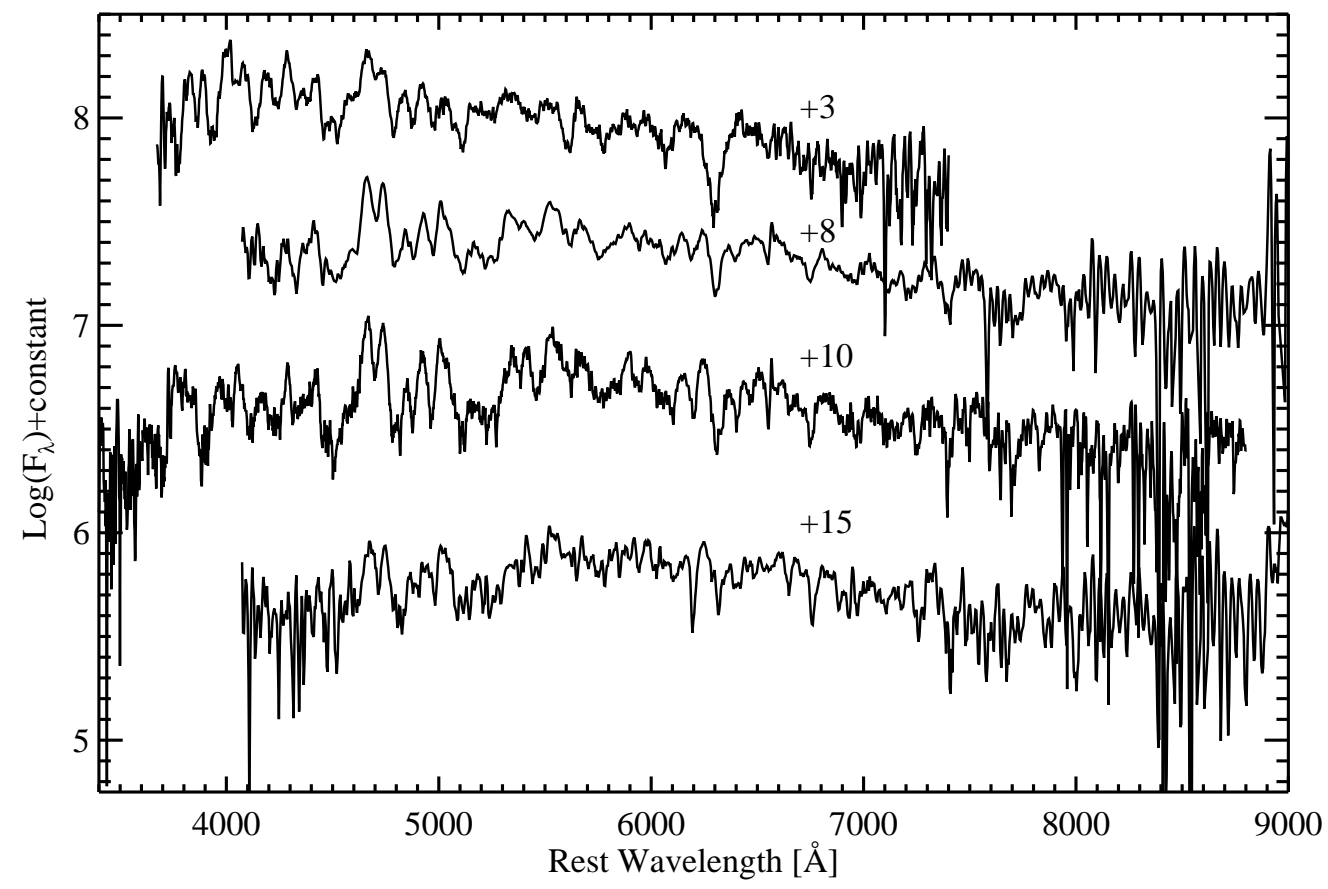

Fig. 5.- Temporal evolution of the spectrum of $07 \mathrm{qd}$ with a boxcar smoothing of 3 pixels in order to clearly show major features. Times in this figure are given in days past $B$-band maximum, telluric absorption has been removed, and wavelengths have been corrected to the rest frame. No extinction corrections have been applied.

\section{Analysis}

\subsection{Energetics}

We infer a rise time of $10 \pm 2$ days based on the nondetections in the SDSS-II data, much shorter than that of typical SN Ia (Havden et al. 2010). We began our analysis by applying the light-curve fitters MLCS2k2 (Jha et al. 2007) and SALT2 (Guv et al. 2007) to the 07qd, 05hk, 02cx, and 08ha data using the SNANA platform (Kessler et al. 2009b). The resulting fits were of very poor quality, with $\chi^{2}>60$ per degree of freedom, suggesting the inability of these algorithms, trained on normal events, to fit the colors and light-curve shapes of 02cx-like peculiar supernovae.

We decided to take a simple approach and estimate the $B$-band light-curve stretch 
Goldhaber et al. 2001) versus peak brightness for the peculiar events and some more normal SN Ia. We used SNANA (Kessler et al. 2009b) to convert from ugriz to the standard $U B V R I$ for SDSS-II SN Ia with $z<0.12$, verifying the uncertainty with the conversions of Fukugita et al. (1996). SNANA applies the MLCS2k2 K-correction algorithm, which is based on a color-matched normal SN Ia spectral energy distribution. This may introduce small errors due to differences in the spectral features between 07qd and typical SN Ia.

For 07qd we find a maximum absolute magnitude (after correcting only for Milky Way Galaxy extinction) of $M_{B}=-15.4 \pm 0.2 \mathrm{mag}$. We estimate that the time of peak $B$ brightness occurred on MJD $54405 \pm 2$, though the true value could be smaller due to the lack of data on the rise. The slopes of the blue light curves of 07 qd flatten out rapidly after 10 days. Consequently, $\Delta m_{15}(B) \approx 1.5 \mathrm{mag}$ for $07 \mathrm{qd}$, or roughly the same as that of $05 \mathrm{hk}$ (Phillips et al. 2007). As seen in Figure 2, 07qd clearly fades more quickly than does 05hk, suggesting that in this case, the measurement of $\Delta m_{15}(B)$ does not compare well with other SN Ia.

Based on our estimation of the rise time, Figure 6 compares the absolute magnitude of $07 \mathrm{qd}$ and its $B$-band stretch with those of 02cx, 05hk, 08ha, and the normal SDSS-II SN Ia (see Sako et al. 2008 for a list of these SN). The low-redshift set of SDSS-II contains a handful of $91 \mathrm{bg}$-like events with stretch parameters $\sim 0.8$, but $07 \mathrm{qd}$ and 08 ha have narrower light curves and are much fainter. There appears to be a sequence connecting the bright, peculiar events 05hk and 02cx to the extreme 08ha with the intermediate 07qd in Figure 6.

To examine the bolometric luminosities, a blackbody was fit to the ugriz spectral energy distribution (SED) as measured at $B$ maximum brightness. Ultraviolet measurements shortward of $u$ were not available for $07 \mathrm{qd}$. 05hk and 08ha experienced severe photospheric line blanketing at $\lambda<3500 \AA$ (Phillips et al. 2007; Foley et al. 2009), which, along with our Keck spectrum (10 days past maximum), suggests that the luminosity of 07qd drops off sharply at that point. Using a Hubble constant of $H_{0}=73 \mathrm{~km} \mathrm{~s}^{-1} \mathrm{Mpc}^{-1}$ (Freedman et al. 2001; Riess et al. 2009) and our measured redshift, a luminosity distance of $177 \mathrm{Mpc}(m-M=36.2 \mathrm{mag})$ was computed. Integrating our SED (corrected for Milky Way extinction) and applying our luminosity distance, we calculated the peak quasibolometric luminosity to be $(4.4 \pm 0.5) \times 10^{41} \mathrm{ergs} \mathrm{s}^{-1}$. Assuming "Arnett's rule" (Arnett 1982; Arnett, Branch, \& Wheeler 1985) as applied by Stritzinger et al. (2006), we estimate the synthesized ${ }^{56} \mathrm{Ni}$ mass using the ratio of maximum luminosity to the luminosity produced by one solar mass of ${ }^{56} \mathrm{Ni}$,

$$
M_{\mathrm{Ni}}=L_{\max } / E_{\mathrm{Ni}}\left(1 \mathrm{M}_{\odot}\right)=\frac{(4.4 \pm 0.5) \times 10^{41} \mathrm{ergs} \mathrm{s}^{-1}}{\alpha\left(6.45 \mathrm{e}^{-t_{\mathrm{R}} \lambda_{\mathrm{Ni}}}+1.45 \mathrm{e}^{-t_{\mathrm{R}} \lambda_{\mathrm{Co}}}\right) \times 10^{43} \mathrm{ergs} \mathrm{s}^{-1} \mathrm{M}_{\odot}^{-1}},
$$




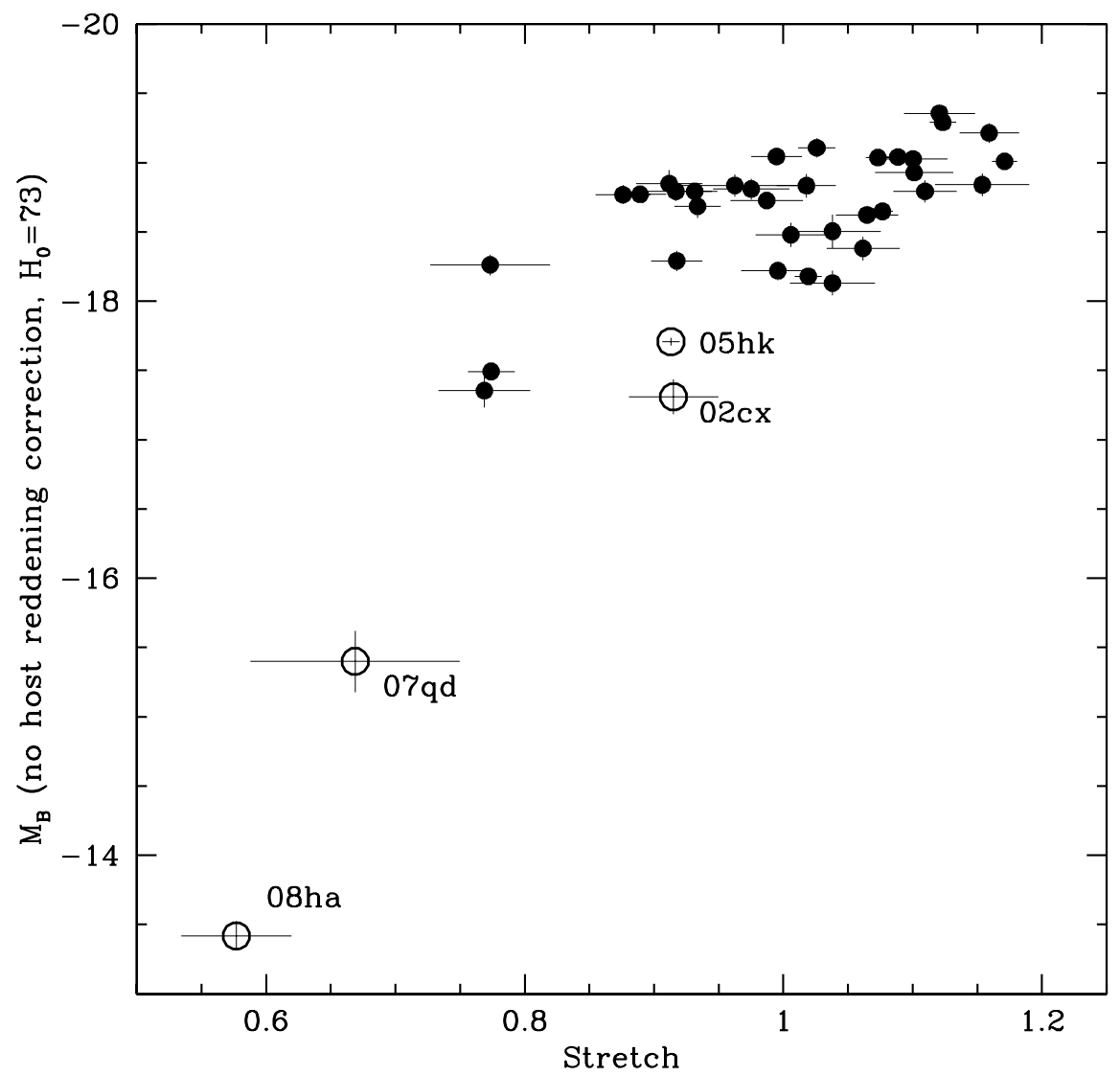

Fig. 6.- Light-curve stretch factors are compared to the absolute magnitudes of peculiar SN Ia (open circles) along with SDSS-II SN Ia at $z<0.12$ (Frieman et al. 2008; Sako et al. 2008). Maximum $B$ for $07 \mathrm{qd}$ is based on an estimated 10-day rise time. No correction for host-galaxy extinction has been made.

where $\lambda_{\mathrm{Ni}}=1 / 8.8$ days and $\lambda_{\mathrm{Co}}=1 / 111.3$ days are the e-folding times for ${ }^{56} \mathrm{Ni}$ and ${ }^{56} \mathrm{Co}$, respectively. Thus, for our maximum possible $t_{\mathrm{R}}=10 \pm 2$ day rise time, we estimate an upper limit of $M_{\mathrm{Ni}}=0.013 \pm 0.002 \mathrm{M}_{\odot}$, which is nearly a factor of 5 larger than that of 08ha $\left[(3.0 \pm 0.9) \times 10^{-3} \mathrm{M}_{\odot}\right.$; Foley et al. 2009], but still much smaller than that of $05 \mathrm{hk}$ [ $\sim 0.22 \mathrm{M}_{\odot}$; Phillips et al. 2007]. Here, we assume that the efficiency of radioactive energy deposition $\alpha$ is unity (see discussion in Howell et al. 2009).

The ejecta mass may be estimated by utilizing the treatments of Arnett (1982) and Pinto \& Eastman (2000a, b) , assuming an opacity of $0.1 \mathrm{~cm}^{2} \mathrm{~g}^{-1}$, as per an Fe II spectrum in the same vein as Foley et al. (2009). Comparing 07qd to a typical SN Ia of kinetic energy $10^{51} \mathrm{ergs}$, rise time 17 days (Hayden et al. 2010), and photospheric velocity 10,000 $\mathrm{km} \mathrm{s}^{-1}$, we derived an ejecta mass of $0.15 \mathrm{M}_{\odot}$. This could be even lower if the rise time of $07 \mathrm{qd}$ is under 10 days or using higher estimates of standard SN Ia rise times. Based on a photospheric 
velocity 3 days past maximum brightness of $2800 \mathrm{~km} \mathrm{~s}^{-1}$ (see $\S 3.2 .1$ ), this translates to a kinetic energy of $\sim 2.0 \times 10^{49} \mathrm{ergs}$. This is similar to the kinetic-energy estimate of 08ha by Foley et al. (2010), but roughly an order of magnitude lower than the $\sim 8 \times 10^{50}$ ergs of 05hk based on its photospheric velocity (Phillips et al. 2007). Typical SN Ia contain ${ }^{56} \mathrm{Ni}$ masses between 0.1 and $1.0 \mathrm{M}_{\odot}$ and ejecta masses ranging from $0.5 \mathrm{M}_{\odot}$ for $\mathrm{SN} 1991 \mathrm{bg}$ to the canonical $1.4 \mathrm{M}_{\odot}$ (Stritzinger et al. 2006). Both of these values are significantly larger than our findings for $07 \mathrm{qd}$.

\subsection{Spectroscopy}

We used SYNOW (Fisher et al. 1997; Fisher 2000), a parameterized supernova spectrum synthesis code, to fit our spectra to profiles of various ions at specific velocities, excitation temperatures, and opacities. These ions are simulated in an expanding photosphere of a chosen blackbody temperature, and the resulting spectra can be compared with data. We systematically fit our spectra with ions commonly seen in SN Ia at similar epochs (Fe II, Co II, Si II; see Hatano et al. 1999 and Maeda et al. 2010 for lists of expected ions and isotopic yields), and attempted to simulate hydrogen and helium to rule out the possibility of a Type II or Ib SN. The photospheric velocity was fixed at each epoch by the best fit for the Fe II lines, and subsequent elements were fit either residing in that photosphere or at detached velocities. After confirming the presence or absence of these species, we tried to fit elements uncommon to normal SN Ia in order to fit any remaining line profiles.

We also attempted to use Na I D host and interstellar absorption to measure dust extinction though the procedures of Munari \& Zwitter (1997). The low signal-to-noise ratio of our spectra, combined with features at various velocities scattered throughout the continuum, created too much uncertainty in these equivalent widths to fix an extinction value.

\subsection{1. +3 Days Spectrum}

Figure 7 and Table 3 present the results of our SYNOW fit to the spectrum 3 days after maximum brightness, as well as a decomposition of the fit to show the influence of each ion. We found from the Fe II lines a photospheric velocity of $2800 \mathrm{~km} \mathrm{~s}^{-1}$, which is extraordinarily low. Typical SN Ia photospheric velocities at this epoch are often in excess of 10,000 $\mathrm{km} \mathrm{s}^{-1}$ (Pskovskii 1977; Branch et al. 1981). The low velocities found for most of the regions makes it highly unlikely that the strong feature at $6300 \AA$ is from hydrogen or helium either in the photosphere or detached. The best fit to the $6300 \AA$ absorption was 
Si II, since the velocity of Fe II was constrained when fitting the $\sim 6100 \AA, \sim 6200 \AA$, and $\sim 6400 \AA$ features as well as others in the bluer spectral regions. Si II fit the absorption best when at a velocity of $800 \mathrm{~km} \mathrm{~s}^{-1}$ lower than that of the iron-group ions. Also prominent in the $\sim 6100 \AA$ region is a broad primary O I line. The secondary signatures of O I are weakly detected at $\sim 5300 \AA$, but masked at $\sim 6400 \AA$ due to the strong Si II feature nearby.

Si III and C III were implemented in a manner similar to that used by Chornock et al. (2006) to help Co II shape the area around $\sim 4600 \AA$. C II, also present in the maximum-light spectrum of 08ha (Foley et al. 2010), was necessary for an absorption feature at $\sim 6550 \AA$. Our SYNOW fits suggest the presence of Mg I, Ti I, Cr I, Ca II, and Al I. Hatano et al. (1999) do not predict these species in a thermonuclear explosion when Si III is strong. It is likely that the features we attribute to these ions are due to other unidentified species or different velocities for existing ones, though we cannot definitively rule out these unusual identifications. It should also be noted that C I, O II, Ni II, and Co III, predicted by Hatano et al. (1999), may be added with little effect on the overall fit. We were unable to fit the absorption between 3800 and $3900 \AA$ at this photospheric velocity with anything other than K II, though its inclusion would introduce other discrepancies to the fit.

\subsection{2. +10 Days Spectrum}

The SYNOW fit in Figure 8 is derived from parameters used to fit 02cx at 12 days past maximum (Branch et al. 2004), except the velocities and blackbody temperature have been reduced to accommodate the unique nature of $07 \mathrm{qd}$. This fit was chosen due to its consistency in representing 08ha, 02cx, and 05hk at similar epochs, as is seen in Figure 9. The similarity is especially apparent with 08ha, which shares most absorption features with $07 \mathrm{qd}$. The relative shift between these two spectra was measured to be $\sim 800 \mathrm{~km} \mathrm{~s}^{-1}$; the photospheric velocity of 08ha at this epoch is $2000 \mathrm{~km} \mathrm{~s}^{-1}$, while the best-fit SYNOW photospheric velocity of $07 \mathrm{qd}$ is $\sim 2800 \mathrm{~km} \mathrm{~s}^{-1}$ with a blackbody temperature of $8000 \mathrm{~K}$. Though Foley et al. (2009) found an excellent match to the 14-day spectrum of 08ha with a low photospheric velocity of only $600 \mathrm{~km} \mathrm{~s}^{-1}$, we attempted our fits with the Fe II estimated velocity of $2000 \mathrm{~km} \mathrm{~s}^{-1}$.

Much of the continuum is dominated by narrow Fe II lines, with Co II playing a large role below $5000 \AA$ as well, consistent with most iron-core SN (Harkness et al. 1991). The modified SYNOW fit also continues to include a prominent Si II feature at $6300 \AA$ necessitated by the inability of Fe II to fit it without losing other features. This Si II resides within the photosphere and enhances the spectrum at $\sim 4100 \AA$, but less so at $\sim 3850 \AA$ and $\sim$ $5025 \AA$. The carbon and vanadium measured at 3 days past maximum are not prominent 


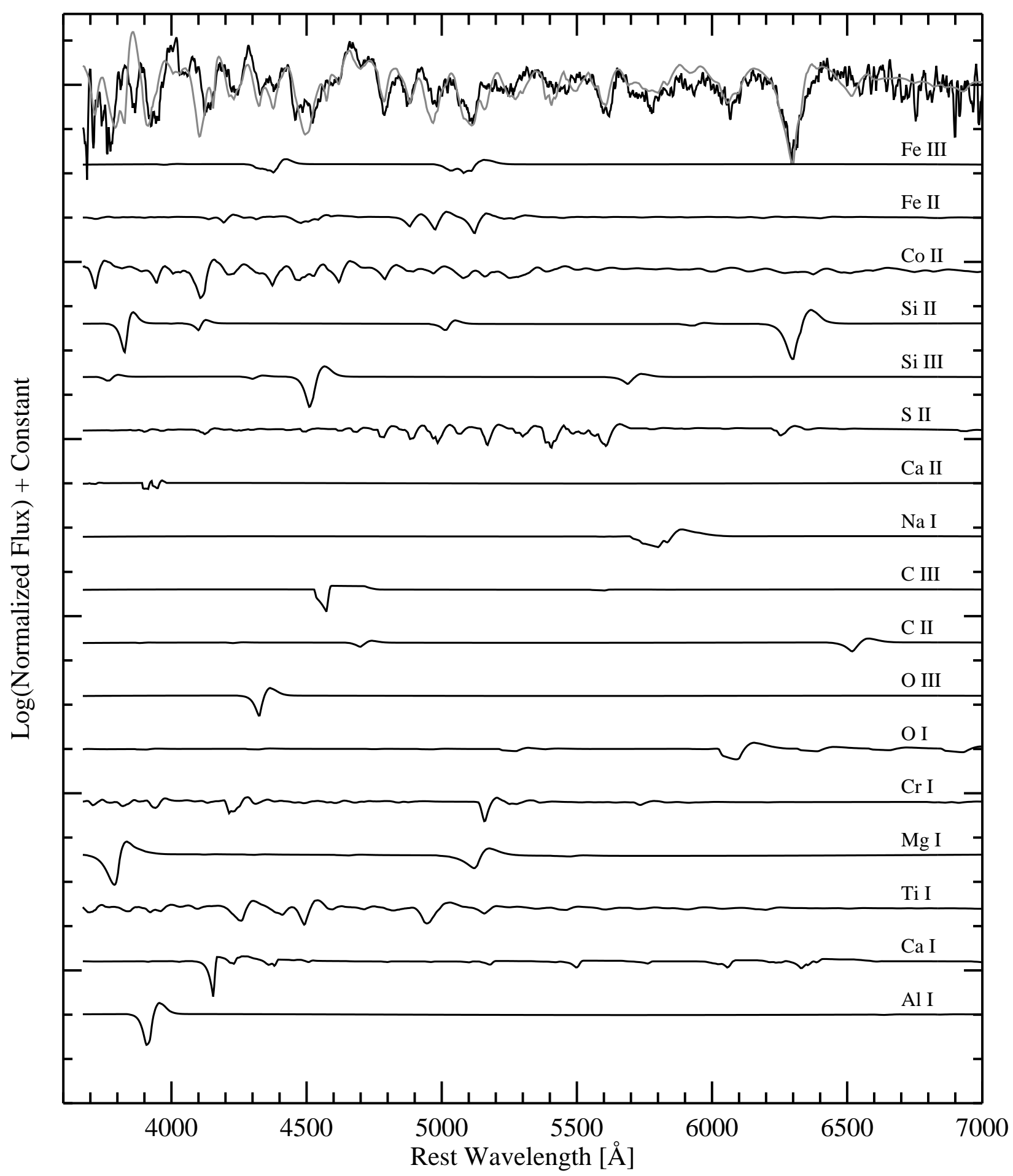

Fig. 7.- The spectrum of 07qd at 3 days past maximum (top) and the best SYNOW fit (gray line). The contribution of each individual species is also shown. All spectra have been normalized, while the data and combined fit have been scaled up by a factor of 2 for clarity. Our more confident identifications are presented near the top while lines at the bottom are less likely. 


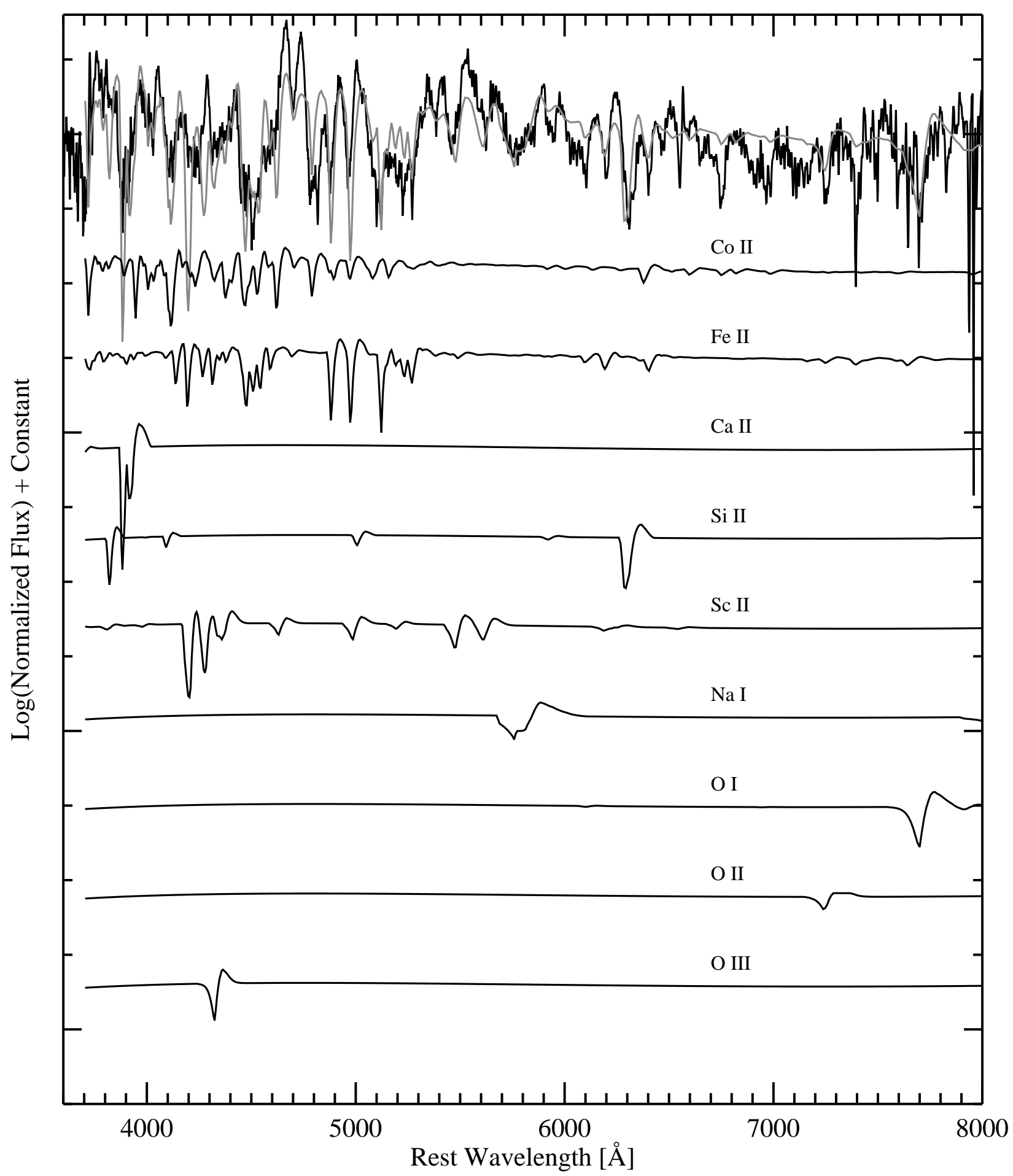

Fig. 8. - Normalized 07qd spectrum 10 days after maximum, with SYNOW fit and its constituents. Again, the data and combined fit have been scaled up by a factor of 2 for clarity, and species are listed roughly in order of decreasing confidence. 


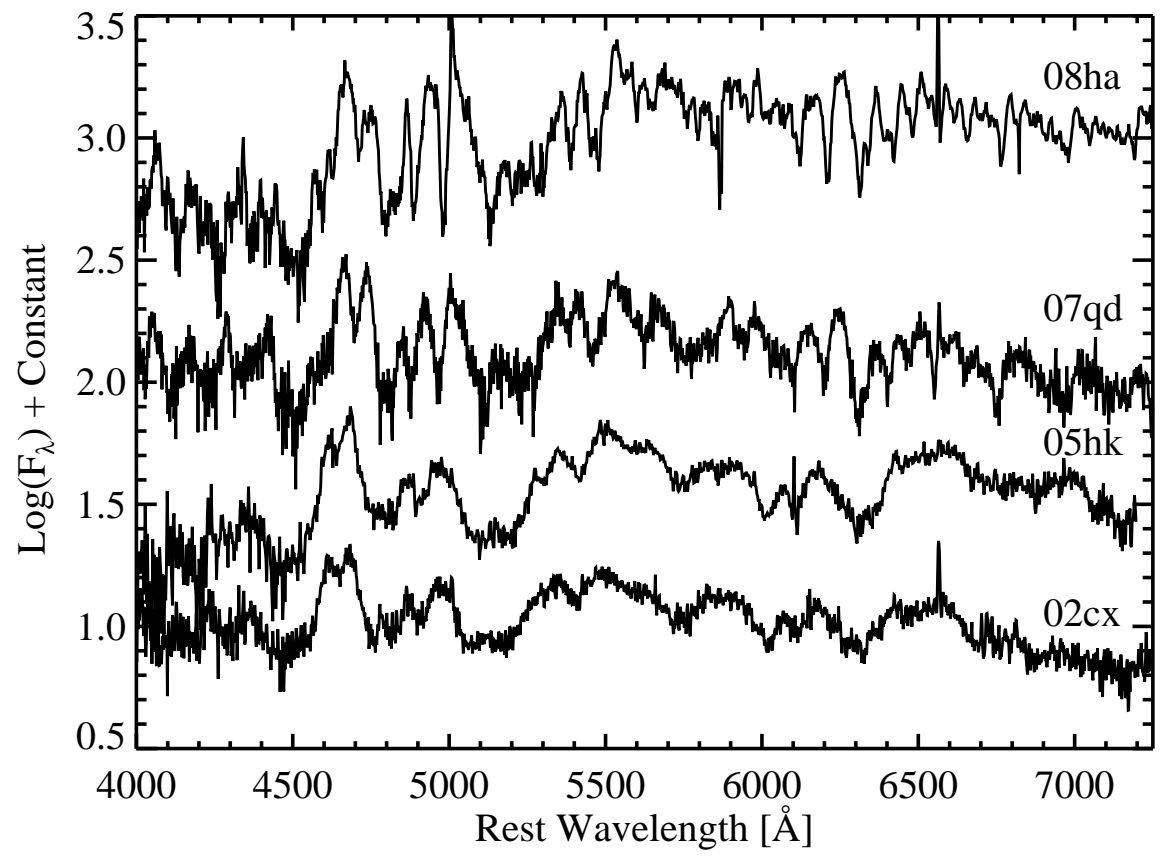

Fig. 9.- Comparison of the spectrum of $07 \mathrm{qd}$ at 10 days past maximum brightness with that of 08ha (Foley et al. 2009), 05hk (Phillips et al. 2007), and 02cx (Branch et al. 2004) at similar epochs (10 days for 08ha, and 12 days for both $05 \mathrm{hk}$ and $02 \mathrm{cx}$ ). All spectra have been appropriately shifted to their rest frames. Each feature of 07qd is redward of those of $05 \mathrm{hk}$ and $02 \mathrm{cx}$, indicating significantly slower photospheric velocities, while only slightly blueward of those of 08ha.

at any ionization stage. Their existence cannot be ruled out, in part due to the influence of the dominant Fe II lines (Baron, Lentz, \& Hauschildt 2003). The Cr II in the 02cx fit by Branch et al. (2004) is also not necessary, as there is insufficient data to examine regions below $3750 \AA$ at this and later epochs. We were unable to identify any ionization stage of $\mathrm{Ti}$ at this epoch, despite the possibility of observing Ti I at 3 days past maximum. Foley et al. (2009) identified Ti II at the same epoch in 08ha, but its addition here does little to the overall fit. Other ions include Sc II (for the absorption at $5450 \AA$ ) and Ca II (for the H\&K lines). The secondary absorption lines S II are no longer apparent at this epoch, as Sc II is able to effectively represent the 5400-5700 $\AA$ region without aid.

The signal-to-noise ratio of the data appears to be lower than it actually is, due to the low-velocity nature of this particular photosphere. Much of the absorption blending in the regions blueward of $4500 \AA$ proved difficult to fit with SYNOW models, especially where P-Cygni profiles are no longer apparent; it is suspected to be a consequence of the narrow absorption features of several iron-group elements. 
$\mathrm{H} \alpha$ and helium ions were tested in SYNOW fits, though no match could be found. We expect and observe host-galaxy $\mathrm{H} \alpha$ emission at $6563 \AA$, though just blueward of this feature we see a small absorption that we identify with C II. The nature of the trough at $\sim 6750 \AA$ is unclear at this time; very few ions are capable of modeling it without severely affecting the fit elsewhere. He I was of particular interest in the SYNOW fit to the 08ha spectrum 13 days past maximum conducted by Valenti et al. (2009) and to SN 2007J, another 02cx-like SN (Foley et al. 2009), but the Na I D line dominates over the predicted He I line. At 10 days past maximum, all other He I features fail to match the spectrum.

\subsubsection{Spectral Evolution and Similarity to Other SN Ia}

Using these SYNOW fits to the individual epochs, the spectral evolution of 07qd given in Figure 5 provides a detailed picture of a developing photosphere. Examining the contributions near $\sim 4550$ and $\sim 5200 \AA$ present at 3 days but absent at 10 days, Fe III has either greatly fallen in opacity or recombined into Fe II, which has increased in influence. Na I and S II have also decreased in intensity, but remain crucial to the region between 5000 and $6000 \AA$.

The Si II feature endures through two weeks past maximum, but its strength has lessened, becoming roughly equal to that of Fe II by day 15 . Much of the spectrum in the blue region was not measured at subsequent epochs, though extended red wavelengths are given, revealing probable O I and O II signatures. It is apparent, however, that the early-time spectra beyond $8000 \AA$ demonstrate the presence of the Ca II near-IR feature, though the Ca II H\&K near-UV lines persist. Blackbody temperatures have fallen to $8000 \mathrm{~K}$ at 8 days past maximum and to $6000 \mathrm{~K}$ at 13 days, further intensifying the influence of Fe II profiles over the continuum. It remains to be seen whether the other unidentified line profiles can be remedied with more exotic ions.

Figure 9 shows the spectra of four 02cx-like SN Ia compared at similar epochs. 07qd clearly bears the strongest resemblance to 08ha; very few features differ. 02cx and 05hk exhibit much faster photospheres than 07qd and 08ha, and are likewise shifted toward the blue. The faster photosphere and higher excitation temperatures used in the SYNOW fit of 02cx (Branch et al. 2004) broaden many of the Fe II lines, effectively masking the primary Si II absorption, but other major velocity features remain consistent. It should be noted, however, that SYNOW's highly parameterized fitting routine permits many degrees of freedom.

In order to independently identify these IMEs outside of models, we directly compared 
spectra of 07qd with those of other confirmed SN Ia. Of special interest is the spectral relation of 07qd to SN 2004eo (hereafter 04eo; Pastorello et al. 2007). Similar to the prototypical SN 1992A (Hamuy et al. 1996c), 04eo is characterized as a fast-declining SN Ia with slow photospheric velocities. However, it retains an absolute $B$ maximum of -19.08 mag (within the range of "normal" SN Ia) and still fits the Phillips (1993) relation. Spectroscopically, 04eo contains strong Si II and S II lines, typical of SN Ia. When the spectra of 07qd are blueshifted to coincide with the photospheric velocity of $04 \mathrm{eo}$, many common features become apparent, especially the Si II and S II lines. Figure 10 shows a normalized comparison between earlytime spectra of $07 \mathrm{qd}$ and $04 \mathrm{eo}$ that highlights both the presence and the relative strengths of these lines.

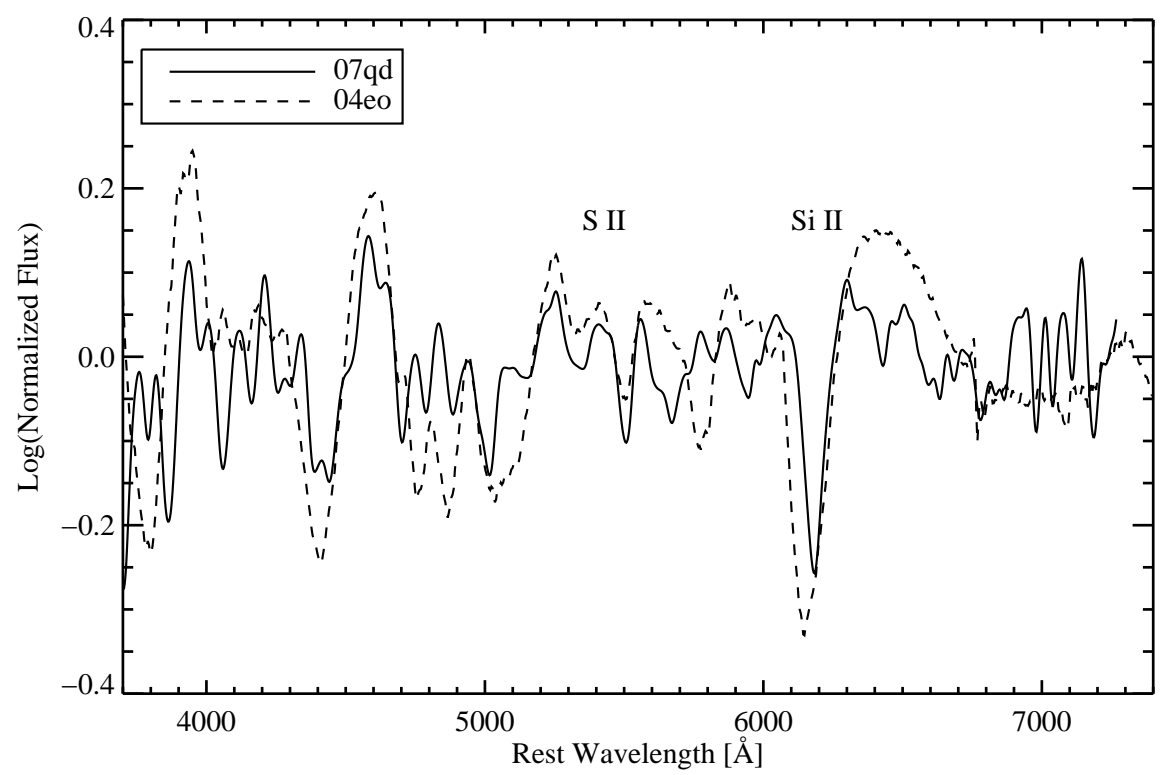

Fig. 10.- Normalized spectrum of 07qd compared with that of SN Ia 2004eo (Pastorello et al. 2007) and shown in the rest frame. The day 3 spectrum of $07 \mathrm{qd}$ has been convolved with a $30 \AA$ FWHM Gaussian and blueshifted by $5500 \mathrm{~km} \mathrm{~s}^{-1}$ to emphasize the similar Si II and S II features.

Similarly, we may compare the maximum-light spectra of 07qd, 08ha, and 05hk, as seen in Figure 11. The spectra of both 07qd and 08ha, with photospheres blueshifted by 2000 $\mathrm{km} \mathrm{s}^{-1}$ and $1750 \mathrm{~km} \mathrm{~s}^{-1}$ (respectively), mirror many of the major features. Although the Si II and S II profiles are noticeably stronger in $07 \mathrm{qd}$ and $08 \mathrm{ha}$, their presence in $05 \mathrm{hk}$ is clear. The similarity between $07 \mathrm{qd}$ and $05 \mathrm{hk}$ below $5000 \AA$ is striking — identical structures in this region are indicative of similar iron-group contributions.

The greatly different photospheric velocities apparent in 07qd and 05hk are examined along with 02cx and 08ha in Figure 12, suggesting a relation between the luminosities and 


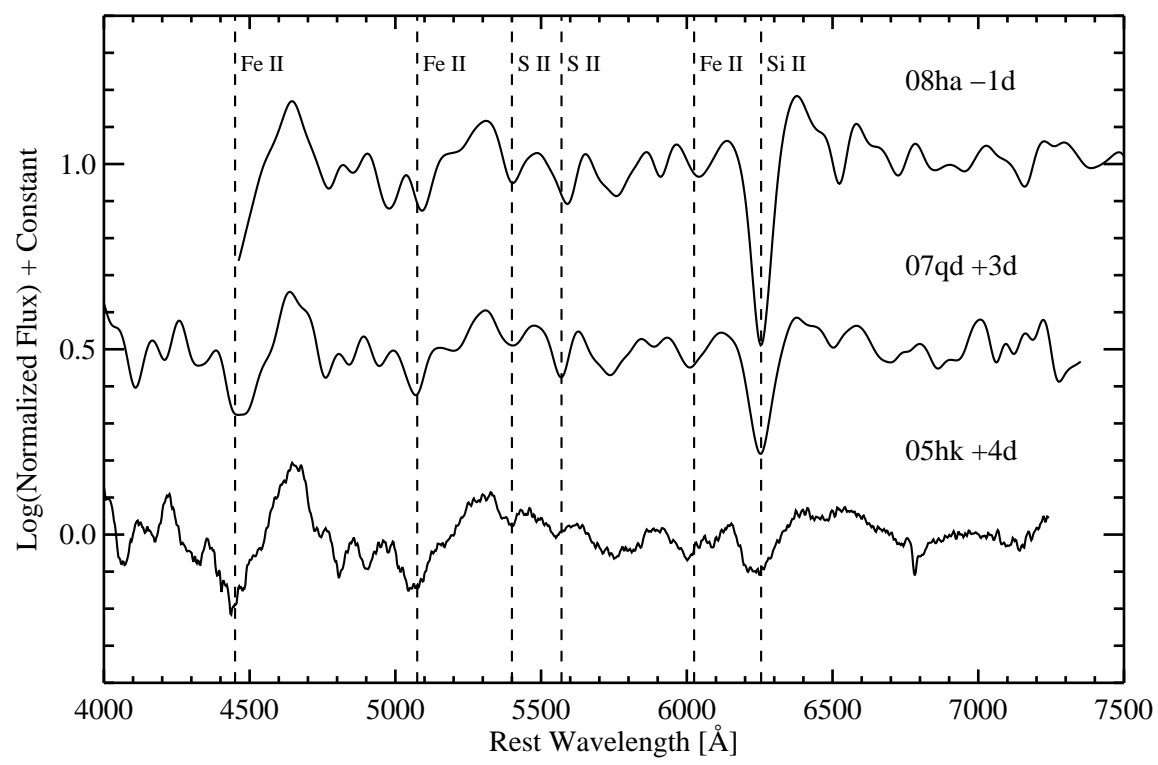

Fig. 11. - Normalized spectra of peculiar SN Ia, observed near maximum brightness. The spectra of $07 \mathrm{qd}$ and 08 ha have been blueshifted by 2000 and $1750 \mathrm{~km} \mathrm{~s}^{-1}$, respectively, to roughly match the photospheric velocity of $05 \mathrm{hk} .07 \mathrm{qd}$ and $08 \mathrm{ha}$ have also been convolved by $50 \AA$ FWHM Gaussians, while 05hk was smoothed with a $5 \AA$ FWHM Gaussian.

expansion velocity in these peculiar cases. We used the Fourier cross-correlation subroutine (FXCOR) in IRAF to determine the average offset of the similar features in these spectra, namely the Fe II and Si II lines. The average photospheric velocities of 07qd are faster than those of 08ha at all epochs when both have measured spectra, but lower than those of any other 02cx-like event. We compare at 10-12 days past maximum because it is here that we have spectra of all four of these 02cx-like SN. To first order, we extrapolated the photospheric velocity of $07 \mathrm{qd}$ at maximum to be roughly $200 \mathrm{~km} \mathrm{~s}^{-1}$ greater than that of $08 \mathrm{ha}$, implying a larger kinetic energy by a factor of 1.14 (assuming the same ejected masses for both SN). Since our calculation in $\S 3.1$ assumes nickel mass is directly related to luminosity and varies with the rise time by a $\sim 7 \%$ change in ${ }^{56} \mathrm{Ni}$ mass per day, the rise-time dependence is small. As a result, the plot of the photospheric velocity versus peak luminosity suggests that the ${ }^{56} \mathrm{Ni}$ yield (via maximum brightness and rise time) and kinetic energy per unit mass (via photospheric velocity) are correlated in 02cx-like events.

Through FXCOR, the average rate of decline in the photospheric velocity is found to be $93 \pm 15 \mathrm{~km} \mathrm{~s}^{-1}$ day $^{-1}$ for the $6355 \AA$ Si II line and $70 \pm 21 \mathrm{~km} \mathrm{~s}^{-1}$ day $^{-1}$ for the other absorption features (dominated by Fe II). Both of these values group the velocity evolution of 07qd amongst the faint SN Ia described by Benetti et al. (2005), and they are smaller in magnitude than those of $08 \mathrm{ha}\left(142 \pm 26 \mathrm{~km} \mathrm{~s}^{-1}\right.$ day $^{-1}$ for Si II and $110 \pm 10 \mathrm{~km} \mathrm{~s}^{-1}$ day $^{-1}$ for 
all other ions). It is difficult to determine the Si II velocities after maximum brightness for 05hk and 02cx since they are quickly blended with Fe II lines. Phillips et al. (2007) found for both $05 \mathrm{hk}$ and $02 \mathrm{cx}$ that the velocities of the $4555 \AA$ Fe II line and the Ca II H\&K lines remained constant at roughly $6000 \mathrm{~km} \mathrm{~s}^{-1}$ for several weeks, implying that the overall expansions were quite slow even for typical SN Ia. If we assume a constant deceleration through late times, the $700 \mathrm{~km} \mathrm{~s}^{-1} \mathrm{Fe}$ II lines found 227 days past maximum in 02cx imply a $23 \mathrm{~km} \mathrm{~s}^{-1}$ day $^{-1}$ decline (Jha et al. 2006).

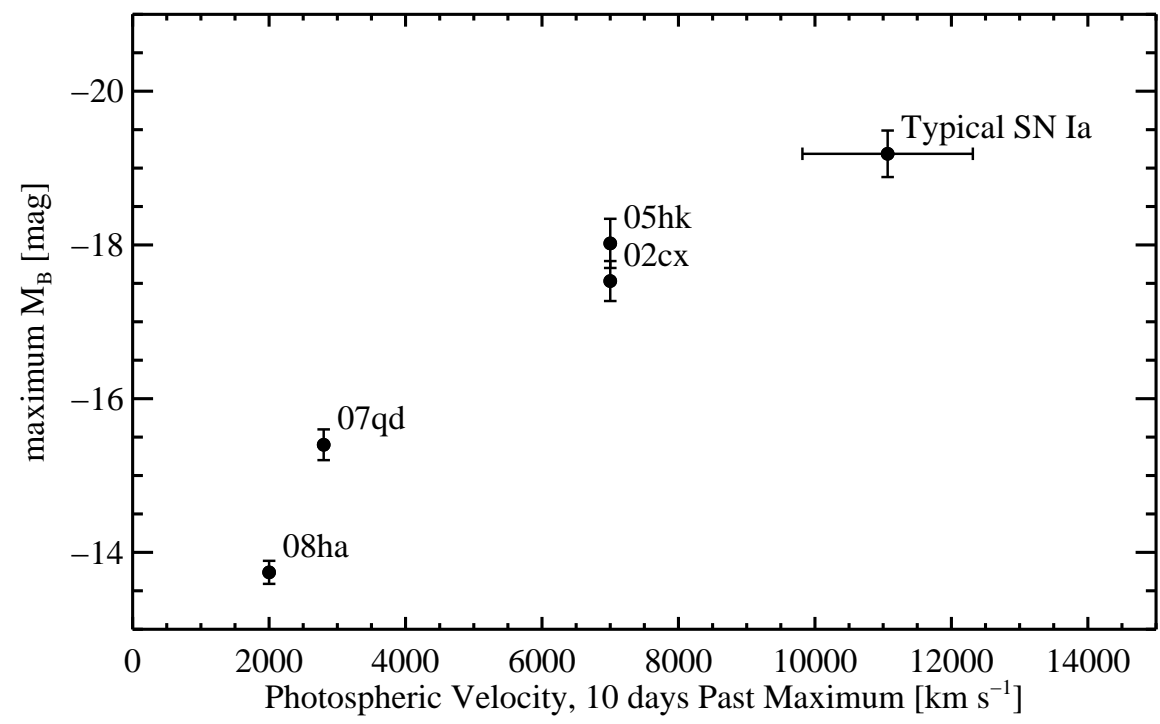

Fig. 12.- Estimated photospheric velocities at $\sim 10$ days past maximum brightness are referenced by their maximum absolute magnitude (based on a $\sim 10$ day rise time for $07 \mathrm{qd}$ ). The "typical" point in the upper-right corner is the root-mean square maximum $B$ magnitude and velocity of the normal SN Ia found by Benetti et al. (2005).

\subsection{Host Galaxy}

The host galaxy in Figure 1 shows a prominent bar and two major spiral arms. The low inclination allows minor spiral arms to be distinguishable, one of which contains the location of 07qd. The classifications of $\mathrm{SBb}$ or $\mathrm{SBc}$ suggest ongoing star formation, but 02cx-like SN Ia appear to span a wide variety of galactic morphologies (Foley et al. 2009; Valenti et al. 2009). The Keck-I spectrum also included the host galaxy's core in the long slit, allowing a measurement of the oxygen abundance. We used both the optimized method recommended in Kewley \& Dopita (2002) and the [N II]/[O II] diagnostic to arrive at an oxygen abundance in the host galaxy of $12+\log (\mathrm{O} / \mathrm{H})=8.79 \pm 0.03$. This is equivalent to 
the Sun's oxygen abundance of $8.76 \pm 0.07$ (Caffau et al. 2008), but it exceeds that of the host galaxy of 08ha, measured to be $8.16 \pm 0.15$ by Foley et al. (2009) using N2 and O3N2 diagnostics (see Pettini \& Pagel 2004).

\section{Discussion}

The striking similarity of the +10 day (relative to $B$ maximum) spectrum of $07 \mathrm{qd}$ to that of 08ha suggest that they are indeed similar explosions. Since 07qd is also spectroscopically linked to 05hk and thus to 02cx as well (see Figure 11), these four peculiar events range in peak luminosity by $4 \mathrm{mag}$, but constitute a single spectral class.

Valenti et al. (2009) argued that 08ha and possibly other 02cx-like objects are actually core-collapse SN; their low luminosity is the result of either the collapse of a $\gtrsim 30 \mathrm{M}_{\odot}$ star directly to a black hole, or electron capture in the core of a 7-8 $\mathrm{M}_{\odot}$ star. However, the clear presence of IMEs (notably Si II $\lambda 6355$ and S II $\lambda \lambda 5968,6359$ ) in 07qd suggests low-density thermonuclear burning and not a core-collapse SN Ib or SN Ic explosion (see Filippenko 1997 for a review of SN spectroscopic classification). Except for the photospheric velocities, the spectra of 07qd are similar to those of SN 1992A-like SN Ia, further strengthening the arguments that it is a thermonuclear runaway.

The presence of these IMEs mirrors the findings of Folev et al. (2010). SN Ibc have been observed with traces of Si II (Valenti et al. 2008) or S II (Nomoto et al. 2000; Brown et al. 2007). However, these lines are quite strong in $07 \mathrm{qd}$, suggesting that it is a SN Ia rather than a core-collapse event. Additionally, the light curve of $07 \mathrm{qd}$, though displaying a sharp rise that is unusual for a typical SN Ia, does not show the fast decline typical of a lowluminosity SN Ic or the "plateau" of a SN IIP that achieves similar peak luminosity. The energy released by $07 \mathrm{qd}$ is substantially lower than that of normal SN Ia, but the predicted nickel mass serves as an intermediate example between 08ha and 05hk, further linking the two.

When compared with other 02cx-like SN, spectra of 07qd mirror that of 08ha and display striking relationships to others. These comparisons also imply that strong Si II is possible in $02 \mathrm{cx}$ and $05 \mathrm{hk}$, though "disguised" by Fe II blending due to their higher photospheric velocities. The fast evolution of 07qd suggests that these IMEs are only detectable for a brief time, and become masked by Fe II or recombine as the photosphere slows and cools. The presence of carbon and oxygen ions in the photosphere echoes the results of deflagration models including those of Gamezo et al. (2004), suggesting the presence of unburned white dwarf material and supporting that this class stems from such a progenitor. 
Also of interest is the "SN .Ia" model, which concerns doubly degenerate white dwarf pairs undergoing a helium flash strong enough to produce ${ }^{56} \mathrm{Ni}$ and absolute $V$ magnitudes as low as $-15 \mathrm{mag}$ (Bildsten et al. 2007). Shen et al. (2010) used this model to predict the luminosities and rise times that we observe, but also spectra dominated by the Heburning products $\mathrm{Ca}$ and $\mathrm{Ti}$ (detected but not prominent). Combined with the low observed expansion velocities, we find $07 q d$ to be an unlikely SN .Ia candidate.

Our estimated radioactive nickel yield for 07qd $\left(\sim 0.01 \mathrm{M}_{\odot}\right)$ is extremely small, and it is difficult to understand how such a weak thermonuclear explosion could disrupt a Chandrasekhar-mass white dwarf. Woosley et al. (2007) found that a minimum of $4.6 \times$ $10^{50}$ ergs of nuclear energy is required to unbind a Chandrasekhar-mass white dwarf, and that can be achieved by producing $0.3 \mathrm{M}_{\odot}$ of ${ }^{56} \mathrm{Ni}$. This amount is more than ten times the ${ }^{56} \mathrm{Ni}$ yield we estimate for $07 \mathrm{qd}$. Of course, nonradioactive elements might dominate the nuclear energy term; for example, the production of $0.37 \mathrm{M}_{\odot}$ of IMEs could also disrupt the star. However, the synthesized elements in three-dimensional pure-deflagration models discussed by Blinnikov et al. (2006) are dominated by the iron peak, especially ${ }^{56} \mathrm{Ni}$, over IMEs. It is also possible that the synthesis of nonradioactive ${ }^{58} \mathrm{Ni}$ or other stable iron-group elements may supply at least some of the kinetic energy.

To estimate the nickel yield, we have applied "Arnett's rule" (Arnett 1982), which allows us to use the peak luminosity as an indicator of the radioactive energy deposition. The rule assumes that nearly all of the energy produced by nickel and cobalt decays is trapped and converted to ultraviolet, optical, and infrared radiation. Calculations by Pinto \& Eastman (2000a) indicate that complete trapping is an excellent approximation for the first month after the explosion when the nickel distribution is concentrated toward the center.

Deflagration models suggest that Rayleigh-Taylor instabilities cause hot nickel bubbles to rise as cooler unburned material sinks. Blinnikov et al. (2006) find fairly uniform distributions of ${ }^{56} \mathrm{Ni}$ in radially averaged three-dimensional pure-deflagration simulations. This leaves more radioactive nickel in the outer layers of the ejecta than predicted in detonation models and implies that some of the radioactive energy will be lost. Additionally, Calder et al. (2004) suggest that highly asymmetric deflagrations could both unbind a white dwarf and provide the mechanism through which heavy iron-group elements may be transported to the surface without a detonation.

The ejected mass estimated for 07qd is very low, as is the case for 08ha (Foley et al. 2009, 2010), perhaps suggesting a sub-Chandrasekhar mass explosion. However, even normal SN Ia events such as SN 1992A (Cappellaro et al. 1997; Stritzinger et al. 2006) appear to have ejected less than a Chandrasekhar mass of material based on similar assumptions. 91bg-like events also give significantly low ejected masses from the methods currently being 
applied. We suspect that some important physics is being ignored in the ejected mass calculations and the results should be considered ambiguous.

The presence of cobalt and high photospheric temperatures in the early-time spectra support the conjecture that radioactive elements were mixed up to the outer layers of $07 \mathrm{qd}$. But Pinto \& Eastman (2000a) and Jeffery (1999) show that a uniform ${ }^{56} \mathrm{Ni}$ distribution should only result in a $10 \%$ to $30 \%$ error in the nickel-mass estimate from Arnett's rule, not the order of magnitude needed to reconcile the energetics. An "inverted" SN Ia, where most of the nickel is in the outer layers of the ejecta (assume Jeffery $1999 q$ values are $<1 / 3$ ), would result in inefficient $\gamma$-ray deposition and extreme violation of Arnett's rule. Such a ${ }^{56} \mathrm{Ni}$ distribution, however, is likely to result in the ejecta turning optically thin to $\gamma$-rays very early.

Stritzinger et al. (2006) used the characteristic time for SN Ia to become optically thin to $\gamma$-rays, $t_{0}$, as a way to estimate the ejected mass and kinetic energy. They found that $t_{0}$ ranges between 25 and 35 days for normal SN Ia, coinciding with a range of ejecta and nickel masses. Jeffery (1999) showed that for a fixed ejecta mass, opacity, and density structure, $t_{0} \approx q^{1 / 2}$, so pushing radioactive nickel away from the center (smaller nickel concentration parameter $q$ ) will shorten the time it takes for the ejecta to become optically thin. Figure 13 shows the quasi-bolometric luminosity curve (constructed from ugriz photometry) for 07qd and displays radioactive energy deposition curves parameterized by the fiducial time, $t_{0}$. For high optical depths, the curves should obey Arnett's rule and pass through the light-curve maximum. The models that become optically thin to $\gamma$-rays soon after explosion have the freedom to be shifted to higher nickel yields as the radioactive energy deposition will match the luminosity after maximum light. The best match to the observations is for $t_{0}>40$ days, suggesting the ejecta are efficiently trapping radioactive energy a month after maximum brightness. The optically thin curves decline much faster than the data, implying that Arnett's rule applies even for this weak explosion. 02cx and 05hk, measured more than 200 days past maximum, continued to exhibit narrow P-Cygni profiles in their spectra (Jha et al. 2006; Sahu et al. 2008). 08ha appears to be consistent with this (Foley et al. 2010), so 07qd may be relatively opaque to optical photons and $\gamma$-rays for some time.

\subsection{Conclusions}

Analyzing the photometry and spectroscopy of 07qd, we find the following.

(1) 07qd was spectroscopically similar to both 08ha and 05hk. Strong lines of Fe II and Co II are present in spectra of all three objects, while Fe III and IME features are most 


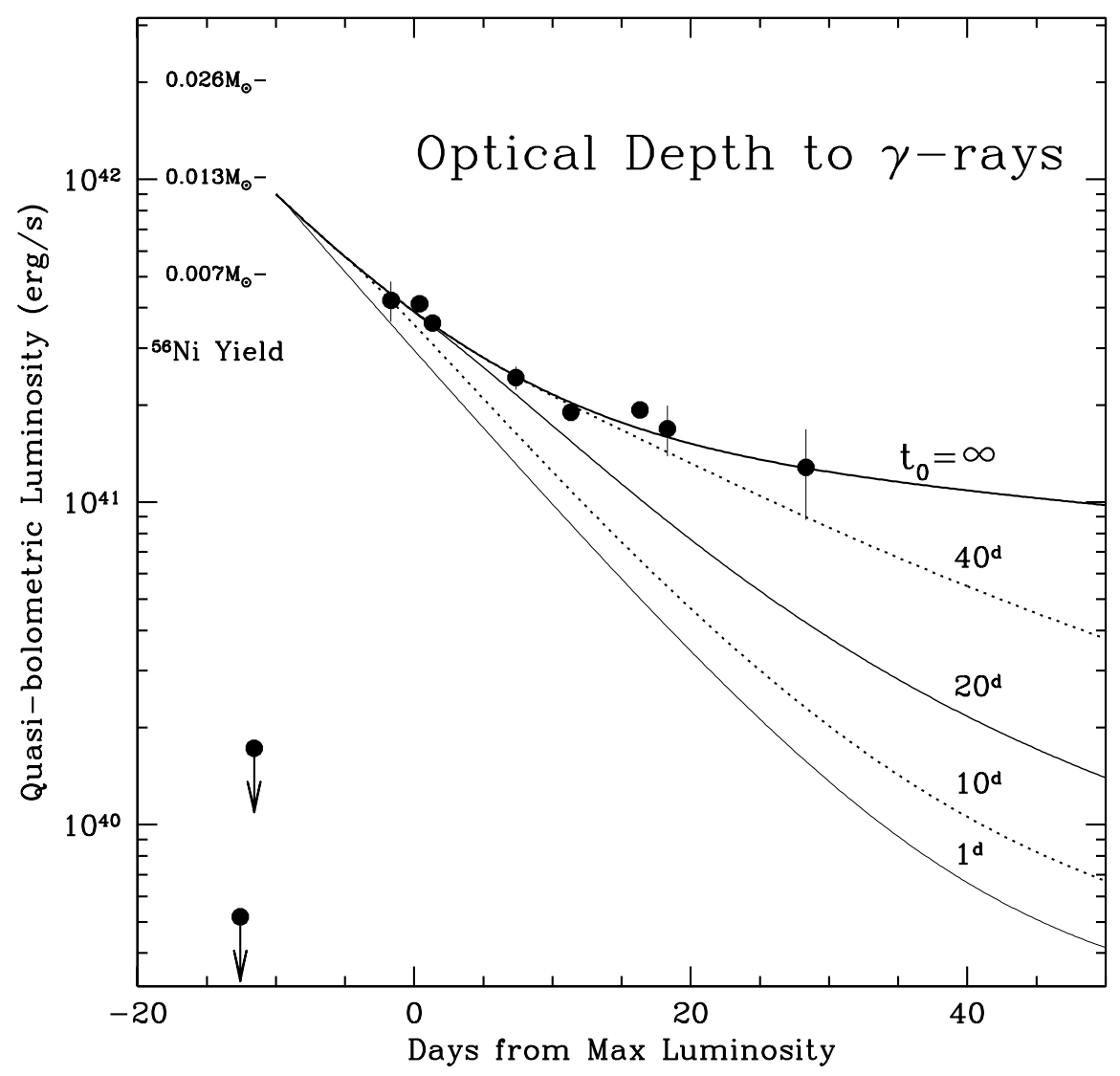

Fig. 13.- The quasi-bolometric luminosity of 07qd estimated from the SDSS-II ugriz photometry versus days from maximum light. The arrows indicate luminosity upper limits from nondetections. The lines show models of radioactive energy deposition which vary in their time to become optically thin to $\gamma$-rays (Jeffery 1999). The models fade faster than the observations for $t_{0}<40$ days, implying that the $\gamma$-ray trapping is efficient for a month after maximum light, similar to the fiducial time for normal SN Ia.

visible at early epochs, though their optical depths decline quickly.

(2) The explicit presence of a variety of IMEs in the early-time spectra implies the thermonuclear deflagration of carbon and oxygen and shows 07qd to be inconsistent with a core-collapse event.

(3) Correlations exist between 02cx-like peak luminosity, photospheric velocity, and light-curve stretch, and the events span a sequence from $08 \mathrm{ha}$ to $02 \mathrm{cx}$.

(4) The diminutive peak luminosity of $07 q d$ implies that the ${ }^{56} \mathrm{Ni}$ yield was quite small, lying between that of $08 \mathrm{ha}$ and $05 \mathrm{hk}$.

Findings (1) and (2) point toward thermonuclear burning during the explosion of 07qd, 
while points (3) and (4) emphasize that 07qd completes a sequence of 02cx-like SN Ia both energetically and spectroscopically. The composition and velocity of the ejecta support the picture of a deflagration. However, deflagration models such as 1_3_3 used by Blinnikov et al. (2006) do not predict the short rise time and small nickel mass seen in 07qd or 08ha. The small amounts of synthesized radioactive nickel argue against the unbinding of a Chandrasekhar-mass progenitor, but if the fusion is dominated by IMEs at the expense of nickel, the explosions of massive white dwarfs are still possible. Other possible scenarios are as follows.

(a) The explosions are thermonuclear with sub-Chandrasekhar masses. This would decrease the progenitors' gravitational binding energies, thus lowering the ejecta masses and internal energies needed to disrupt the stars.

(b) Arnett's rule does not apply to this class of objects, implying that the ${ }^{56} \mathrm{Ni}$ mass is significantly underestimated. The bolometric light curve of $07 \mathrm{qd}$, however, suggests that Arnett's rule is operational to some degree.

(c) The explosions are actually core-collapse scenarios that have somehow managed to synthesize significant amounts of $\mathrm{Si}$ and $\mathrm{S}$.

The low velocities and energies present in 08ha and 07qd enable the analysis of many aspects of 02cx-like SN, otherwise hidden by the Fe II blending present in 05hk and 02cx. Though the velocities and energies span a wide range, together they constitute a well-defined group of peculiar SN Ia.

Funding for the SDSS and SDSS-II has been provided by the Alfred P. Sloan Foundation, the Participating Institutions, the National Science Foundation (NSF), the U.S. Department of Energy (DOE), the National Aeronautics and Space Administration (NASA), the Japanese Monbukagakusho, the Max Planck Society, and the Higher Education Funding Council for England. The SDSS Web Site is http://www.sdss.org/.

The SDSS is managed by the Astrophysical Research Consortium for the Participating Institutions. The Participating Institutions are the American Museum of Natural History, Astrophysical Institute Potsdam, University of Basel, University of Cambridge, Case Western Reserve University, University of Chicago, Drexel University, Fermilab, the Institute for Advanced Study, the Japan Participation Group, Johns Hopkins University, the Joint Institute for Nuclear Astrophysics, the Kavli Institute for Particle Astrophysics and Cosmology, the Korean Scientist Group, the Chinese Academy of Sciences (LAMOST), Los Alamos National Laboratory, the Max-Planck-Institute for Astronomy (MPIA), the Max-Planck-Institute for Astrophysics (MPA), New Mexico State University, Ohio State University, University of 
Pittsburgh, University of Portsmouth, Princeton University, the United States Naval Observatory, and the University of Washington.

Some of the data presented herein were obtained at the W. M. Keck Observatory, which is operated as a scientific partnership among the California Institute of Technology, the University of California, and NASA; it was made possible by the generous financial support of the W. M. Keck Foundation. The authors wish to recognize and acknowledge the very significant cultural role and reverence that the summit of Mauna Kea has always had within the indigenous Hawaiian community; we are most fortunate to have the opportunity to conduct observations from this mountain. We thank the Keck staff for their assistance.

We are grateful for the financial support of the University of Notre Dame, NASA/STScI Grant HST-GO-10893.01-A to C.M.M., the NSF, and the DOE, specifically NSF grant AST0908886 and DOE grant DE-FG02-08ER41563 to A.V.F. Support for this research at Rutgers University was provided by DOE grant DE-FG02-08ER41562 and NSF award AST-0847157 to S.W.J. We thank Brian Hayden, Joe Gallagher, and Weidong Li for discussions and their

help in the production of this paper, Jerod Parrent and the Online Supernova Spectrum Archive (SUSPECT), and David Jeffery along with the SUSPEND database.

\section{REFERENCES}

Adelman-McCarthy, J. K., et al. 2008, ApJS, 175, 297

Aldering, G., et al. 2006, ApJ, 650, 510

Arnett, W. D. 1982, ApJ, 253, 785

Arnett, W. D., Branch, D., \& Wheeler, J. C. 1985, Nature, 314, 337

Baron, E., Lentz, E. J., \& Hauschildt, P. H. 2003, ApJ, 588, L29

Bassett, B., et al. 2007, CBET, 1137

Benetti, S., Cappellaro, E., Turatto, M., Taubenberger, S., Harutyunyan, A., \& Valenti, S. 2006, ApJ, 653, L129

Benetti, S., et al. 2005, ApJ, 623, 1011

Bildsten, L., Shen, K. J., Weinberg, N. N., \& Nelemans, G. 2007, ApJ, 662, L95

Blinnikov, S. I., Röpke, F. K., Sorokina, E. I., Gieseler, M., Reinecke, M., Travaglio, C., Hillebrandt, W., \& Stritzinger, M. 2006, A\&A, 453, 229 
Branch, D. 1981, ApJ, 248, 1076

Branch, D., Baron, E., Thomas, R. C., Kasen, D., Li, W., \& Filippenko, A. V. 2004, PASP, 116, 903

Brown, G. E., Lee, C.-H., \& Moreno Méndez, E. 2007, ApJ, 671, L41

Caffau, E., Ludwig, H.-G., Steffen, M., Ayres, T. R., Bonifacio, P., Cayrel, R., Freytag, B., \& Plez, B. 2008, A\&A, 488, 1031

Calder, A. C., Plewa, T., Vladimirova, N., Lamb, D. Q., \& Truran, J. W. 2004, arXiv:astro-ph/0405162

Cappellaro, E., Mazzali, P. A., Benetti, S., Danziger, I. J., Turatto, M., della Valle, M., \& Patat, F. 1997, A\&A, 328, 203

Cardelli, J. A., Clayton, G. C., \& Mathis, J. S. 1989, ApJ, 345, 245

Chornock, R., Filippenko, A. V., Branch, D., Foley, R. J., Jha, S., \& Li, W. 2006, PASP, 118,722

Filippenko, A. V. 1997, ARA\&A, 35, 309

Filippenko, A. V. 2003, in From Twilight to Highlight: The Physics of Supernovae, ed. W. Hillebrandt \& B. Leibundgut (Berlin: Springer-Verlag), 171

Filippenko, A. V., et al. 1992a, AJ, 104, 1543

Filippenko, A. V., et al. 1992b, ApJ, 384, L15

Fisher, A., Branch, D., Nugent, P., \& Baron, E. 1997, ApJ, 481, L89

Fisher, A. 2000, PhD. Thesis, University of Oklahoma

Foley, R. J., et al. 2003, PASP, 115, 1220

Foley, R. J., et al. 2009, AJ, 138, 376

Foley, R. J., Brown, P. J., Rest, A., Challis, P. J., Kirshner, R. P., \& Wood-Vasey, W. M. 2010, ApJ, 708, L61

Freedman, W. L., et al. 2001, ApJ, 553, 47

Frieman, J. A., et al. 2008, AJ, 135, 338 
Fukugita, M., et al. 1996, AJ, 111, 1748

Gamezo, V. N., Khokhlov, A. M., \& Oran, E. S. 2004, in Cosmic Explosions in Three Dimensions, ed. P. Höflich, P. Kumar, \& J. C. Wheeler (Cambridge: Cambridge Univ. Press), 121

Garnavich, P. M., et al. 1998, ApJ, 493, L53

Garnavich, P. M., et al. 2004, ApJ, 613, 1120

Goldhaber, G., et al. 2001, ApJ, 558, 359

Gunn, J. E., et al. 1998, AJ, 116, 3040

Gunn, J. E., et al. 2006, AJ, 131, 2332

Guy, J., et al. 2007, A\&A, 466, 11

Hamuy, M., Phillips, M. M., Maza, J., Suntzeff, N. B., Schommer, R. A., \& Avilés, R. 1995, AJ, 109, 1

Hamuy, M., Phillips, M. M., Suntzeff, N. B., Schommer, R. A., Maza, J., Smith, R. C., Lira, P., \& Aviles, R. 1996, AJ, 112, 2438

Hamuy, M., et al. 1996a, AJ, 112, 2391

Hamuy, M., et al. 1996b, AJ, 112, 2398

Hamuy, M., et al. 2003, Nature, 424, 651

Harkness, R. P. 1991, in SN 1987A and Other Supernovae, ed. I. J. Danziger \& K. Kjär (Garching: ESO), 447

Hatano, K., Branch, D., Fisher, A., Millard, J., \& Baron, E. 1999, ApJS, 121, 233

Hayden, B. T., et al. 2010, submitted (arXiv:1001.3428)

Hill, G. J., et al. 1998, SPIE Conf., 3355, p. 424

Holtzman, J. A., et al. 2008, AJ, 136, 2306

Horne, K. 1986, PASP, 98, 609

Howell, D. A., et al. 2009, ApJ, 691, 661

Jeffery, D. J. 1999, arXiv:astro-ph/9907015 
Jha, S., Riess, A. G., \& Kirshner, R. P. 2007, ApJ, 659, 122

Jha, S., et al. 2004, AJ, 132, 189

Kasen, D. 2006, ApJ, 649, 939

Kewley, L. J., \& Dopita, M. A. 2002, ApJS, 142, 35

Kessler, R., et al. 2009a, ApJS, 185, 32

Kessler, R., et al. 2009b, PASP, 121, 1028

Khokhlov, A. M. 1991, A\&A, 245, 114

Lampeitl, H., et al. 2009, MNRAS, 1782

Leibundgut, B., et al. 1993, AJ, 105, 301

Leonard, D. C., et al. 2002, AJ, 124, 2490

Li, W., et al. 2003, PASP, 115, 453

Livio, M. 2000, in Type Ia Supernovae: Observations and Theory, ed. J. C. Niemeyer \& J. W. Truran (Cambridge: Cambridge Univ. Press), 33

Lupton, R. H., Gunn, J. E., \& Szalay, A. S. 1999, AJ, 118, 1406

Maeda, K., Roepke, F. K., Fink, M., Hillebrandt, W., Travaglio, C., \& Thielemann, F. K. 2010, submitted (arXiv:1002.2153)

Munari, U., \& Zwitter, T. 1997, A\&A, 318, 269

Nomoto, K., et al. 2000, in Gamma-ray Bursts, 5th Huntsville Symposium, 526, 622

Nugent, P., Phillips, M., Baron, E., Branch, D., \& Hauschildt, P. 1995, ApJ, 455, L147

Oke, J. B., et al. 1995, PASP, 107, 375

Pastorello, A., et al. 2007, MNRAS, 377, 1531

Perlmutter, S., et al. 1999, ApJ, 517, 565

Pettini, M., \& Pagel, B. E. J. 2004, MNRAS, 348, L59

Phillips, M. M. 1993, ApJ, 413, L105

Phillips, M. M., et al. 1992, AJ, 103, 1632 
Phillips, M. M., et al. 1999, AJ, 118, 1766

Phillips, M. M., et al. 2007, PASP, 119, 360

Pinto, P. A., \& Eastman, R. G. 2000a, ApJ, 530, 744

Pinto, P. A., \& Eastman, R. G. 2000b, ApJ, 530, 757

Prieto, J., Garnavich, P., Depoy, D., Marshall, J., Eastman, J., \& Frank, S. 2005, CBET, 302,1

Pskovskii, Yu. P. 1977, Sov. Astron. 21, 675

Reinecke, M., Hillebrandt, W., \& Niemeyer, J. C. 2002, A\&A, 391, 1167

Richmond, M. W., et al. 1996, AJ, 111, 327

Riess, A. G., Press, W. H., \& Kirshner, R. P. 1995, ApJ, 438, L17

Riess, A. G., Press, W. H., \& Kirshner, R. P. 1996, ApJ, 473, 88

Riess, A. G., et al. 1998, AJ, 116, 1009

Riess, A. G., et al. 2009, 699, 539

Sahu, D. K., et al. 2008, ApJ, 680, 580

Sako, M., et al. 2008, AJ, 135, 348

Sauer, D. N., Mazzali, P. A., Deng, J., Valenti, S., Nomoto, K., \& Filippenko, A. V. 2006, MNRAS, 369, 1939

Schlegel, D. J., Finkbeiner, D. P., \& Davis, M. 1998, ApJ, 500, 525

Schmidt, W., \& Niemeyer, J. C. 2006, A\&A, 446, 627

Shen, K. J., Kasen, D., Weinberg, N. N., Bildsten, L., \& Scannapieco, E. 2010, arXiv:1002.2258

Smith, J. A., et al. 2002, AJ, 123, 2121

Sollerman, J., et al. 2009, ApJ, 703, 1374

Stritzinger, M., et al. 2006, A\&A, 450, 241

Travaglio, C., Hillebrandt, W., Reinecke, M., \& Thielemann, F.-K. 2004, A\&A, 425, 1029 
Turatto, M., et al. 1996, MNRAS, 283, 1

Valenti, S., et al. 2008, ApJ, 673, L155

Valenti, S., et al. 2009, Nature, 459, 674

Wade, R. A., \& Horne, K. 1988, ApJ, 324, 411

Woosley, S. E., Kasen, D., Blinnikov, S., \& Sorokina, E. 2007, ApJ, 662, 487

Yokoo, T., Arimoto, J., Matsumoto, K., Takahashi, A., \& Sadakane, K. 1994, PASJ, 46, L191

York, D. G., et al. 2000, AJ, 120, 1579 
Table 1. Observed SDSS Photometry of 07qd in Flux Densities ${ }^{\mathrm{a}, b}$

\begin{tabular}{cccccc}
\hline \hline MJD & $u[\mu \mathrm{Jy}]$ & $g$ & $r$ & $i$ & $z$ \\
\hline 54346.41 & $-0.010 \pm 2.726$ & $1.620 \pm 1.230$ & $0.880 \pm 1.235$ & $-0.730 \pm 1.281$ & $-1.100 \pm 3.851$ \\
54348.41 & $-1.780 \pm 1.614$ & $0.610 \pm 0.648$ & $1.520 \pm 0.698$ & $0.200 \pm 1.019$ & $1.600 \pm 3.929$ \\
54355.42 & $1.500 \pm 2.146$ & $-0.470 \pm 0.530$ & $0.410 \pm 1.111$ & $-0.370 \pm 1.813$ & $5.870 \pm 5.868$ \\
54358.37 & $4.310 \pm 1.878$ & $0.870 \pm 0.530$ & $-0.000 \pm 0.707$ & $-1.480 \pm 1.159$ & $-1.940 \pm 4.186$ \\
54365.40 & $0.000 \pm 0.971$ & $-0.180 \pm 0.315$ & $0.000 \pm 0.496$ & $-1.500 \pm 0.719$ & $-1.470 \pm 3.225$ \\
54381.42 & $1.400 \pm 1.165$ & $0.010 \pm 0.362$ & $-0.090 \pm 0.590$ & $-0.340 \pm 0.790$ & $-4.300 \pm 3.010$ \\
54384.43 & $1.550 \pm 1.396$ & $0.130 \pm 0.393$ & $-0.580 \pm 0.557$ & $-2.860 \pm 0.817$ & $-5.780 \pm 3.438$ \\
54386.41 & $0.260 \pm 1.478$ & $0.080 \pm 0.489$ & $0.230 \pm 0.634$ & $0.670 \pm 0.846$ & $-1.960 \pm 4.057$ \\
54388.42 & $0.240 \pm 1.431$ & $0.270 \pm 0.399$ & $0.060 \pm 0.587$ & $-0.250 \pm 0.779$ & $2.090 \pm 3.177$ \\
54392.42 & $-0.840 \pm 1.554$ & $-0.420 \pm 0.556$ & $1.160 \pm 0.837$ & $0.120 \pm 1.228$ & $-1.630 \pm 4.251$ \\
54393.42 & $2.100 \pm 1.731$ & $-0.020 \pm 0.564$ & $-0.670 \pm 0.546$ & $-0.050 \pm 0.850$ & $3.650 \pm 4.373$ \\
54396.29 & $-7.950 \pm 7.556$ & $-2.870 \pm 3.713$ & $0.720 \pm 2.329$ & & - \\
54405.39 & $13.620 \pm 2.416$ & $19.170 \pm 1.007$ & $16.510 \pm 0.990$ & $15.750 \pm 1.281$ & $-3.160 \pm 7.6840 \pm 4.463$ \\
54406.33 & $6.930 \pm 1.466$ & $17.310 \pm 0.638$ & $17.080 \pm 0.709$ & $15.930 \pm 0.912$ & $17.020 \pm 2.989$ \\
54412.35 & $2.470 \pm 1.702$ & $9.760 \pm 0.514$ & $15.720 \pm 0.899$ & $13.850 \pm 1.204$ & $15.080 \pm 4.166$ \\
54416.32 & $0.920 \pm 1.072$ & $6.350 \pm 0.388$ & $14.140 \pm 0.574$ & $13.620 \pm 0.769$ & $17.010 \pm 3.035$ \\
54421.33 & $3.480 \pm 1.736$ & $5.250 \pm 0.502$ & $13.290 \pm 0.797$ & $11.980 \pm 1.110$ & $12.360 \pm 4.757$ \\
54423.31 & $1.060 \pm 1.924$ & $4.200 \pm 0.829$ & $12.460 \pm 1.058$ & $13.620 \pm 1.197$ & $19.620 \pm 3.800$ \\
54433.33 & & $5.430 \pm 1.501$ & $8.780 \pm 2.494$ & $9.740 \pm 2.832$ & $5.980 \pm 6.150$ \\
\hline
\end{tabular}

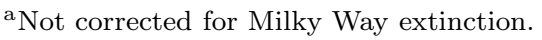

${ }^{b}$ Data associated with poor seeing have been omitted from this list. 
Table 2. Journal of Spectroscopic Observations

\begin{tabular}{cccccc}
\hline \hline Telescope & UT Date & Time [UT] & Days since $B_{\max }$ & Exposure [s] & Range $[\AA]$ \\
\hline TNG & 5 Nov. 2007 & $02: 06: 10$ & +3 & $3 \times 1800$ & $3673-7401$ \\
HET & 10 Nov. 2007 & $04: 23: 12$ & +8 & 1200 & $4075-9586$ \\
Keck & 12 Nov. 2007 & $12: 39: 01$ & +10 & 1500 & $3073-8800$ \\
HET & 17 Nov. 2007 & $03: 58: 29$ & +15 & 1200 & $4074-9586$ \\
\hline
\end{tabular}


Table 3. SYNOW Parameters for Figure $7^{\mathrm{p}, b}$

\begin{tabular}{|c|c|c|c|c|c|}
\hline Ion & $\tau$ & $v_{\min }$ & $v_{\max }$ & $v_{e}$ & $T_{\text {exc }}$ \\
\hline $\mathrm{Na} \mathrm{I}$ & 0.3 & 4.5 & 8.0 & 4 & 10 \\
\hline $\mathrm{Na} \mathrm{I}$ & 0.5 & 2.8 & 4.5 & 1 & 10 \\
\hline $\mathrm{Na} \mathrm{I}$ & 0.1 & 8.0 & 10. & 2 & 10 \\
\hline Ca II & 10 & 2.8 & 3.0 & 2 & 12 \\
\hline S II & 2.7 & 2.8 & 3.3 & 2 & 10 \\
\hline Si II & 3.0 & $2.0^{\mathrm{c}}$ & $\infty$ & 1 & 8 \\
\hline Si III & 2.0 & 2.8 & $\infty$ & 1 & 10 \\
\hline Fe III & 0.7 & 2.8 & $\infty$ & 1 & 10 \\
\hline Fe II & 1.0 & 2.8 & $\infty$ & 1 & 10 \\
\hline Co II & 3.0 & 2.8 & $\infty$ & 1 & 10 \\
\hline C II & 0.02 & 2.8 & $\infty$ & 1 & 10 \\
\hline C III & 0.5 & 5.0 & 8.0 & 2 & 10 \\
\hline O I & 9.5 & 2.8 & 6.5 & 3 & 10 \\
\hline O III & 1.8 & 2.8 & $\infty$ & 1 & 10 \\
\hline $\mathrm{Mg} \mathrm{I}$ & 0.5 & 2.8 & $\infty$ & 2 & 12 \\
\hline Ti I & 1.0 & 2.8 & $\infty$ & 1 & 10 \\
\hline Cr I & 2.0 & 2.8 & 4.0 & 2 & 10 \\
\hline Ca I & 2.0 & 5.0 & $\infty$ & 1 & 10 \\
\hline $\mathrm{Al} \mathrm{I}$ & 3.0 & 2.8 & $\infty$ & 1 & 10 \\
\hline
\end{tabular}

${ }^{\text {a }}$ Photospheric velocity of $2800 \mathrm{~km} \mathrm{~s}^{-1}$ and a blackbody temperature of 10,000 K.

${ }^{\mathrm{b}}$ Velocities are given in units of $1000 \mathrm{~km} \mathrm{~s}^{-1}$ and $T_{\text {exc }}$ values are given in units of $1000 \mathrm{~K}$.

${ }^{\mathrm{c}} \mathrm{Si}$ II simulated individually at $2000 \mathrm{~km} \mathrm{~s}^{-1}$ 
Table 4. SYNOW Parameters for Figure 8,

\begin{tabular}{rccccc}
\hline \hline Ion & $\tau$ & $v_{\min }$ & $v_{\max }$ & $v_{e}$ & $T_{\text {exc }}$ \\
\hline Co II & 10 & 2.8 & 3.8 & 1 & 8 \\
Fe II & 10 & 2.8 & 3.8 & 1 & 8 \\
Ca II & 35 & 2.8 & 4.8 & 1 & 8 \\
Na I & 0.4 & 2.8 & 4.8 & 1 & 8 \\
Na I & 0.3 & 4.0 & 7.0 & 8 & 8 \\
Na I & 0.3 & 7.0 & 11.0 & 3 & 8 \\
Si II & 3.0 & 2.8 & 3.8 & 1 & 8 \\
Sc II & 1.0 & 2.8 & 5.8 & 1 & 10 \\
O I & 1.0 & 2.8 & $\infty$ & 1 & 8 \\
O II & 0.2 & 3.5 & $\infty$ & 1 & 8 \\
O III & 2.0 & 2.8 & $\infty$ & 1 & 8 \\
\hline
\end{tabular}

a Photospheric velocity of $2800 \mathrm{~km} \mathrm{~s}^{-1}$ and a blackbody temperature of $8,000 \mathrm{~K}$.

${ }^{\mathrm{b}}$ Velocities are given in units of $1000 \mathrm{~km} \mathrm{~s}^{-1}$ and $T_{\text {exc }}$ values are given in units of $1000 \mathrm{~K}$. 